\title{
Vollendoskopische Operationen der Wirbelsäule bei Bandscheibenvorfall und Spinalkanalstenose
}

\author{
Sebastian Ruetten
}

\section{Zusammenfassung}

Degenerative Einengungen des Spinalkanals mit Kompression neuraler Anteile entstehen durch knöcherne, diskale, kapsuläre oder ligamentäre Strukturen. Häufigste Ursachen sind Bandscheibenvorfälle und Spinalkanalstenosen. Lenden- und Halswirbelsäule stehen im Vordergrund. Nach Ausschöpfen konservativer Maßnahmen kann ein operatives Vorgehen notwendig werden. Als Standardverfahren im lumbalen Bereich gelten heutzutage bei Bandscheibenvorfall und Spinalkanalstenose die mikrochirurgische, mikroskopisch-assistierte Dekompression, im zervikalen Bereich die mikrochirurgische, mikroskopisch-assistierte ventrale Dekompression und Fusion. Beide Verfahren zeigen gute klinische Ergebnisse, weisen aber dennoch operationsbedingte Probleme auf. Dekompressionen im Bereich der Wirbelsäule müssen unter kontinuierlicher Visualisierung erfolgen und die Möglichkeit der suffizienten Knochenresektion beinhalten. Unter Berücksichtigung dessen erfolgte zusammen mit Entwicklung des lateral transforaminalen und interlaminären Zugangs für die Lendenwirbelsäule sowie des dorsalen und kontralateralen ventralen Zugangs für die Halswirbelsäule die Entwicklung komplett neuer Optiken und Instrumentarien für vollendoskopische Operationen. Im Rahmen der klinischen Validierung wurden die Möglichkeiten und Ergebnisse vergleichbarer etablierter Standardverfahren als Maßstab herangezogen. Die Entwicklung der operativen Zugänge sowie der neuen Stablinsenoptiken und entsprechenden Instrumentarien hat im Bereich der Lendenwirbelsäule die vollendoskopische Operation aller primären und Rezidivbandscheibenvorfälle inner- und außer- halb des Spinalkanals sowie von Spinalkanalstenosen technisch ermöglicht, im Bereich der Halswirbelsäule die Resektion weicher Bandscheibenvorfälle. Die Anwendung der jeweiligen Zugänge ist abhängig von anatomischen und pathologischen Ein- und Ausschlusskriterien. Die klinischen Ergebnisse der Standardverfahren werden erreicht, was als Mindestkriterium bei der Einführung neuer Techniken gelten muss. Unter Berücksichtigung von EBM-Kriterien kann festgestellt werden, dass mittels der entwickelten vollendoskopischen Techniken suffiziente Dekompression bei genannten Indikationen erreicht wird mit reduzierter Traumatisierung, verbesserten Sichtbedingungen und positiver Kostenrelation. Somit können vollendoskopische Operationen heute als Erweiterung und Alternative innerhalb des Gesamtkonzepts der Wirbelsäulenchirurgie eingeordnet werden.

\section{Fully Endoscopic Operations of the Spine in Disc Herniations and Spinal Stenosis}

Degenerative constrictions of the spinal canal with compression of neural elements arise as a result of bony, disc, capsular or ligament structures. The most frequent causes are disc herniations and spinal stenoses. The lumbar and cervical spines are the most prominent sites. After conservative treatment has been exhausted without success, surgical intervention may be necessary. Today, microsurgical, microscopicallyassisted decompression is regarded as the standard procedure for disc herniation and spinal stenosis in the lumbar region, while in the cervical spine microsurgical, microscopically-assisted ventral decompression and fusion are standard. Both procedures demonstrate good clinical results but present problems associated with the operation. Decompressions in the area of the spine must be carried out under continuous visualisation and must include the possibility for adequate bone resection. Taking this into account, completely new endoscopes and instrument sets have been developed for fully endoscopic operations in tandem with the development of the lateral transforaminal and interlaminar approaches to the lumbar spine and the posterior and contralateral anterior approaches to the cervical spine. The possibilities and results of comparable established standard procedures were used as a benchmark in the course of a clinical validation. The development of surgically created approaches and the new rod-lens endoscopes combined with appropriate instrument sets have laid the technical foundations for fully endoscopic operations in the lumbar spine for all primary and recurrent disc herniations inside and outside the spinal canal and for spinal stenoses. These developments have also enabled resection of soft disc herniations in the cervical spine. The use of the relevant approaches depends on anatomic and pathological inclusion and exclusion criteria. The clinical results of standard procedures are equalled, which must be regarded as a minimum criterion for the introduction of new technologies. On the basis of EBM criteria, it can be established that, using the newly developed fully endoscopic techniques, adequate decompression is achieved in the defined indications with reduced traumatisation, improved visibility conditions and positive cost benefits. Today, fully endoscopic operations may be regarded as an expansion and alternative within the overall concept of spinal surgery. 


\section{Einleitung}

Degenerative Einengungen des Spinalkanals mit Kompression neuraler Strukturen entstehen durch knöcherne, diskale, kapsuläre oder ligamentäre Strukturen. Je nach Lokalisation und Ausprägung können sie zu klassischen klinischen Symptomen wie z.B. neurogener Claudicatio, radikulären Schmerzen oder radikulären sowie zentralen neurologischen Defiziten führen. Neben dem möglichen Cauda-equina-Syndrom oder der Myelopathie manifestieren sich die meisten Kompressionssyndrome im Sinne einer radikulären Symptomatik, d.h. einer Symptomatik in den oberen oder unteren Extremitäten. Rücken- oder Nackenschmerzen sind eher den sekundären degenerativen Phänomenen wie z.B. segmentaler Instabilität oder Deformität zuzuschreiben. Es existieren verschiedene Hypothesen, um das Auftreten von Schmerzen bei Verengungen des Spinalkanals zu erklären. Neben der rein mechanischen neuralen Kompression werden vaskuläre, inflammatorische und biomechanische Komponenten diskutiert. Eine eindeutige Korrelation zwischen dem bildgebend sichtbaren Ausmaß der Verengung und klinischen Symptomen besteht nicht $[2,18]$.

Die häufigsten Ursachen für degenerative Einengungen des Spinalkanals mit radikulären Symptomen oder neurogener Claudicatio sind Bandscheibenvorfälle sowie laterale oder zentrale Spinalkanalstenosen (Abb.1). Lenden- und Halswirbelsäule stehen zahlenmäßig eindeutig im Vordergrund.

An der Lenden- und Halswirbelsäule sind die häufigsten Ursachen für Kompression neuraler Strukturen Bandscheibenvorfälle und Spinalkanalstenosen.

\section{Dekompressionsoperationen}

Das Ziel in der Behandlung von Patienten mit klinisch symptomatischen Bandscheibenvorfällen oder Spinalkanalstenosen ist eine suffiziente konservative Therapie. Dennoch kann nach Ausschöpfen dieser Maßnahmen, bei intolerablen Schmerzen oder neurologischen Defiziten ein operatives Vorgehen notwendig werden. In diesem Fall müssen in Abhängigkeit von Pathologie und Symptomen Dekompressionsoperationen, Fusionen oder die Kombination beider Verfahren in Betracht gezogen werden. Zahlreiche operative Techniken der Lenden- und Halswirbelsäule wurden beschrieben
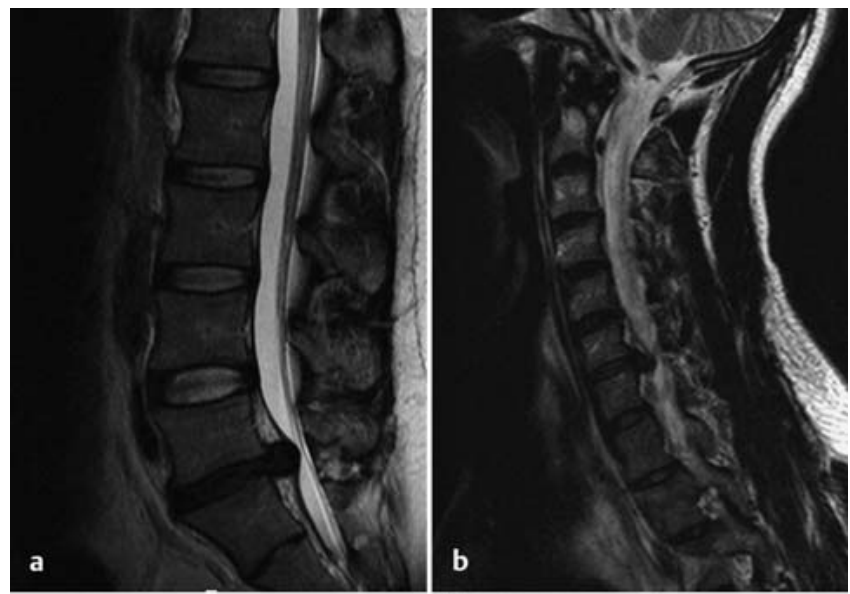

Abb. 1 a bis $d$

Lumbaler (a) und zervikaler (b) Bandscheibenvorfall, lumbale laterale Spinalkanalstenose beidseits mit zusätzlicher Juxtafacettenzyste rechts (c) und lumbale zentrale Spinalkanalstenose (d).

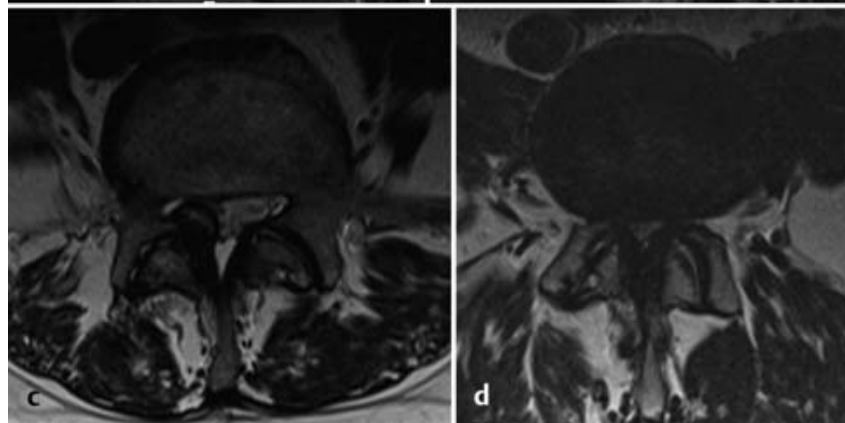

und werden bis heute teilweise kontrovers diskutiert. Insgesamt zeigt sich während der vergangenen Jahre ein Trend weg von eher aggressiven hin zu eher selektiven Verfahren. Derzeit besteht die Tendenz, bei im Vordergrund stehenden radikulären Symptomen ohne eindeutige Anzeichen segmentaler Instabilität und bei Anwendung einer stabilitätserhaltenden Dekompressionstechnik auf die gleichzeitige Fusion zu verzichten. Allerdings existieren keine allgemeingültigen klaren Definitionen über Instabilität und stabilitätserhaltende Techniken.

Unabhängig vom operativen Verfahren müssen zur direkten Dekompression neurale Anteile freigelegt werden. Ein ungehinderter Zugang ist nicht gegeben, da der Spinalkanal schützende Funktion hat und auch durch die komplexen Aufgaben der Wirbelsäule hinsichtlich Stabilität und Mobilität verschlossen ist. Zudem befindet sich die Wirbelsäule nicht an der Körperoberfläche, sondern ist je nach operativem Zugangsweg von verschiedensten anatomischen Strukturen umgeben. Dies bedeutet, dass durch die Dekompression Strukturen des Spinalkanals oder des umgebenden Gewebes geschädigt oder entfernt werden müssen, die für Schutz oder Stabilität wichtig sein können; sei es nur im Rahmen des
Zugangswegs oder weil diese Strukturen selbst Kompressionen mit verursachen.

Bei Dekompressionsoperationen an der Wirbelsäule werden Strukturen traumatisiert oder entfernt, die für Schutz und Stabilität wichtig sein können.

Heutzutage scheint es nach EBM-Kriterien sicher zu sein, dass Dekompressionen radikuläre Symptome und neurogene Claudicatio verbessern können. Das technisch erforderliche Ausmaß der Dekompression und die Bedingungen, unter denen eine zusätzliche Fusion notwendig ist, sind nicht eindeutig geklärt.

\section{Minimalinvasive Dekompressionen}

Konventionelle Dekompression an der Wirbelsäule zeigt gute Resultate. Dennoch treten operationsbedingte Vernarbungen des Epiduralraums auf, die auch klinisch symptomatisch werden können [5]. Zudem erschweren sie erneute Operationen, die an der Wirbelsäule aufgrund des fortschreitenden degenerativen Prozesses nie ausgeschlossen werden können. Vorliegende Studienergebnisse weisen auf die Möglichkeit operationsinduzierter Instabilitäten aufgrund der notwendigen Resektion von Strukturen des Spinalkanals hin [1]. Der Zugangsweg im Innervationsgebiet des 
dorsalen Astes des Spinalnervs kann das stabilisierende und koordinative System negativ beeinflussen [4]. Zugangsbedingte und operationsspezifische Komplikationen und Probleme können auftreten. Die Kombination dieser Parameter kann unbefriedigende Ergebnisse von Revisionsoperationen erklären [7].

Aufgrund dieser Probleme wurde schon von Beginn der Wirbelsäulenchirurgie an versucht, bestehende Operationsverfahren zu modifizieren. Im Vordergrund stehen hierbei Verminderung der Invasivität und Verbesserung der intraoperativen Sicht mit entsprechender Ausleuchtung und Visualisierung der Strukturen. Minimalinvasivere Techniken können Schädigungen des Gewebes und deren Konsequenzen reduzieren $[17,19]$. Diesbez. war der Einsatz der mikrochirurgischen, mikroskopisch-assistierten Technik für Dekompressionsoperationen ein bedeutender Fortschritt und hat sich bis heute zum Standardverfahren etabliert.

Minimalinvasivere Techniken bei Wirbelsäulenoperationen sollen die Traumatisierung reduzieren sowie intraoperative Sicht-und Lichtbedingungen verbessern.

Allgemein haben minimalinvasivere Techniken den Vorteil der reduzierten operationsbedingten Traumatisierung, die sich auch an der Wirbelsäule aufgrund der oben genannten Probleme als vorteilhaft herausgestellt hat. Gleichzeitig kann die intraoperative Visualisierung optimiert werden. Als Nachteil können das reduzierte Arbeitsfeld und damit auch die eingeschränkte Mobilität sowie die anspruchsvolle Operationstechnik mit schwieriger Lernkurve gelten. Je mehr die Traumatisierung reduziert wird, d.h. je minimalinvasiver der Zugang, um so mehr ist die exakte Positionierung im Arbeitsfeld erforderlich, da eine nachträgliche Korrektur des operativen Zugangs eingeschränkt ist.

Ein erfolgreiches Beispiel in der Entwicklung minimalinvasiver Techniken sind endoskopische und endoskopisch-assistierte Operationen, die sich in verschiedenen medizinischen Bereichen bewährt haben. Am Bewegungsapparat zeigen arthroskopische Operationen mit Stablinsenoptiken unter kontinuierlichem Flüssigkeitsstrom die Vorteile der geringeren Invasivität und verbesserten intraoperativen Sicht und übertreffen bei vielen Indikationen die Nachteile.
Entwicklung vollendoskopischer Techniken

Als Ziel in der Entwicklung neuer Operationstechniken muss als Minimum angestrebt werden, klinische Ergebnisse der etablierten Standardverfahren und gleichzeitig Minimierung operationsinduzierter Traumatisierung und deren negativer Langzeitfolgen $\mathrm{zu}$ erreichen. Ein Fokus der technischen Entwicklung in der Wirbelsäulenchirurgie war und bleibt die Optimierung der intraoperativen Sicht- und Lichtbedingungen.

Mit Bezug auf vollendoskopische Verfahren für Wirbelsäulenoperationen erfolgte zunächst an der Lendenwirbelsäule die Entwicklung des lateral transforaminalen und des interlaminären Zugangs. Nachfolgend zeigten sich Probleme auf technischer Seite durch die zur Verfügung stehenden optischen Systeme mit kleinem intraendoskopischen $\mathrm{Ar}$ beitskanal und entsprechend eingeschränktem Instrumentenrepertoire. Es konnten unüberwindbare Schwierigkeiten in der Resektion harten Gewebes, des operativen Zugangswegs sowie der Mobilität entstehen. Das suffiziente $\mathrm{Ar}$ beiten an der Pathologie war technisch limitiert und musste teilweise ohne direkte Visualisierung durchgeführt werden. Die Entwicklung komplett neuer Optiken und Instrumentarien zeigte sich als notwendig. Basierend auf den gewonnenen Erfahrungen wurden hierfür verschiedene Grundvoraussetzungen festgelegt: Stablinsenoptiken für beste optische Qualität; Arbeiten unter kontinuierlichem Flüssigkeitsstrom wie in der Arthroskopie; uniportales Arbeiten im Gegensatz zur Arthroskopie, da im Operationsfeld nicht genügend Raum für 2. Zugang besteht; mit Ausnahme des Zugangs jeder operative Schritt unter kontinuierlicher Visualisierung wie beim konventionellen Operieren; Schaffung eines Operationsraums in einem Gebiet ohne präformierter Höhle.

Nach Definition der maximal notwendigen Instrumentengrößen wurden um den entsprechenden Durchmesser des intraendoskopischen Arbeitskanals die Endoskope entwickelt und nachfolgend die Instrumentarien. Hierdurch wurden die Grundvoraussetzungen erfüllt und die Probleme auf technischer Seite minimiert.

Basierend auf diesen Erfahrungen im Bereich der Lendenwirbelsäule wurde im Bereich der Halswirbelsäule ähnlich vor- gegangen. Für den vollendoskopischen kontralateralen ventralen Zugang musste zusätzlich ein völlig anderes optisches System entwickelt werden. Die Systeme für die Brustwirbelsäule ergaben sich als Letztes aus den bereits entwickelten.

An der Lenden- und Brustwirbelsäule kann vollendoskopisch mittels des trans-/ extraforaminalen oder des interlaminären Zugangs operiert werden, an der Halswirbelsäule mittels des ventralen oder dorsalen Zugangs.

Entwicklungsbegleitend durchgeführte Studien zeigten, dass die Kombination der operativen Zugänge mit den neuen Optiken und Instrumentarien erstmals die Voraussetzungen für ein suffizientes vollendoskopisches Arbeiten schaffte und das Indikationsspektrum erweiterte.

\section{Vollendoskopische Operationen der Lendenwirbelsäule}

\section{Trans-/extraforaminaler Zugang}

Der posterolaterale Zugang für Biopsien aus Wirbelkörpern wurde Ende der 40er-Jahre beschrieben. Perkutane Operationen wurden seit Beginn der 70erJahre angewandt. Endoskope werden seit Beginn der 80er-Jahre eingesetzt, zunächst, um den Intervertebralraum nach beendeter offener Operation zu inspizieren. Hieraus entwickelte sich die endoskopische transforaminale Technik mit posterolateralem Zugang. Diese Technik war die gebräuchlichste in der endoskopischen Operation von Patienten mit Erkrankungen lumbaler Bandscheiben, zeigte aber im Rahmen der Entwicklungsarbeit eindeutige Probleme.

Mit dem posterolateralen transforaminalen Zugang kann der Intervertebralraum innerhalb des Foramen intervertebrale zwischen austretendem und traversierendem Spinalnerv erreicht werden (Abb. 2). Der Eintrittspunkt in der Haut für den operativen Zugang wird standardisiert in Zentimetern von der Mittellinie definiert. Durch Reduktion von intradiskalem Volumen und Druck soll eine bandscheibenbedingte Kompression vermindert werden. Die direkte Entfernung intra- und extraforaminal sequestrierter Bandscheibenanteile ist möglich. Die Entfernung von prolabiertem Bandscheibenmaterial innerhalb des Spinalkanals im Sinne einer retrograden Resektion, d.h. von intradiskal durch den Anulusdefekt, wurde be- 


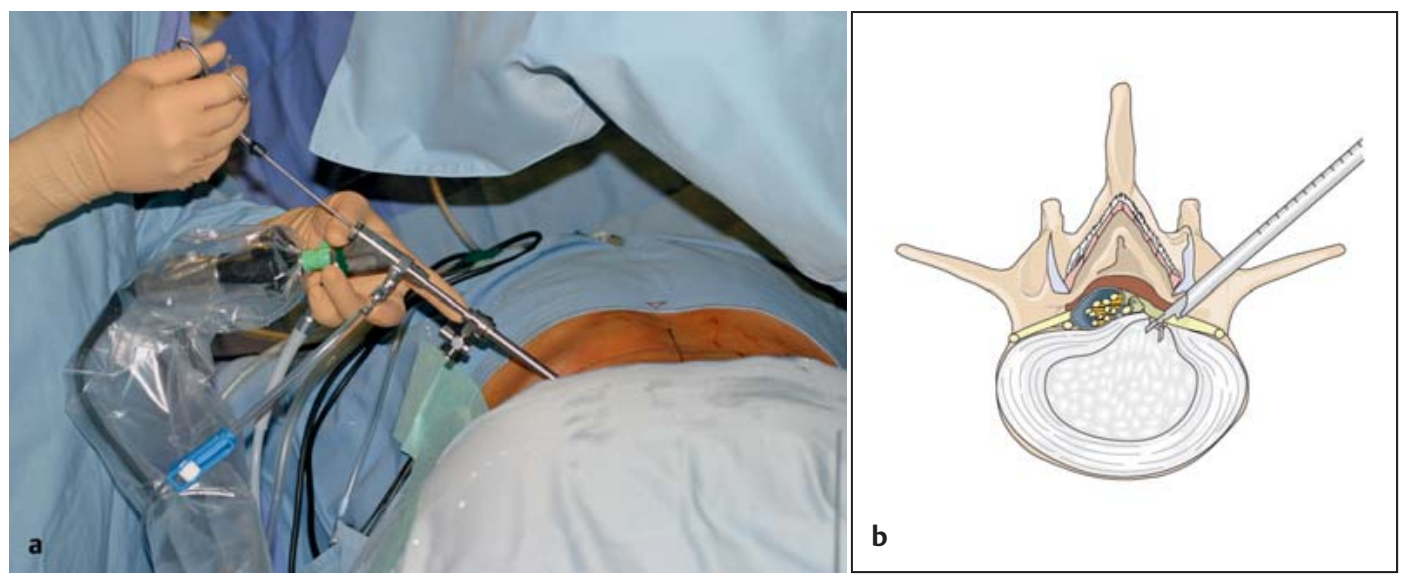

Abb. $2 \mathbf{a}$ und $\mathbf{b}$

Vollendoskopische transforaminale Technik mit posterolateralem Zugang an der Lendenwirbelsäule.

schrieben und als „In-out-Technik“ bezeichnet.

Die häufigste Lokalisation lumbaler Bandscheibenvorfälle betrifft die kaudalen Etagen. Der Durchmesser des lumbalen Foramen intervertebrale nimmt von kranial nach kaudal ab. Eine zusätzliche Einengung des Foramens kann aus kurzen Pedikeln oder degenerativen Veränderungen resultieren. Sequestriertes Nukleusmaterial findet sich innerhalb des Spinalkanals dorsal des Anulus im ventralen Epiduralraum und medial der medialen Pedikellinie. Die klinische Erfahrung zeigt, dass der Anulusdefekt häufig kleiner als der Durchmesser des Sequestervolumens ist. Zusätzlich besteht insbesondere bei dislozierten Sequestern keine kontinuierliche Verbindung nach intradiskal. Bei fortgeschrittener Degeneration besteht der Sequester häufig nicht aus einem zusammenhängenden Stück, sondern kann als körnige Substanz oder einzelne Fragmente vorliegen. In diesen Fällen ist eine Entfernung in einem Stück nicht möglich. Daher ist die beabsichtigte retrograde Resektion von intradiskal häufig technisch nicht möglich. Für eine adäquate Dekompression ist somit das direkte Erreichen des extradiskalen ventralen Epiduralraums unter kontinuierlicher Visualisierung notwendig.

Besonders in den kaudalen Segmenten mit kleinerem Foramen intervertebrale wird diese bei Anwendung des posterolateralen Zugangs verhindert. Somit kann eine suffiziente Dekompression präoperativ nicht eindeutig prognostiziert werden. Auch ein Absenken des Endoskops nach Durchführung des Zugangs zum tangentialen Erreichen des ventralen Spinalkanals wird durch die Vorlaufstrecke im Weichteilgewebe und die Zygoapophysealgelenke verhindert.

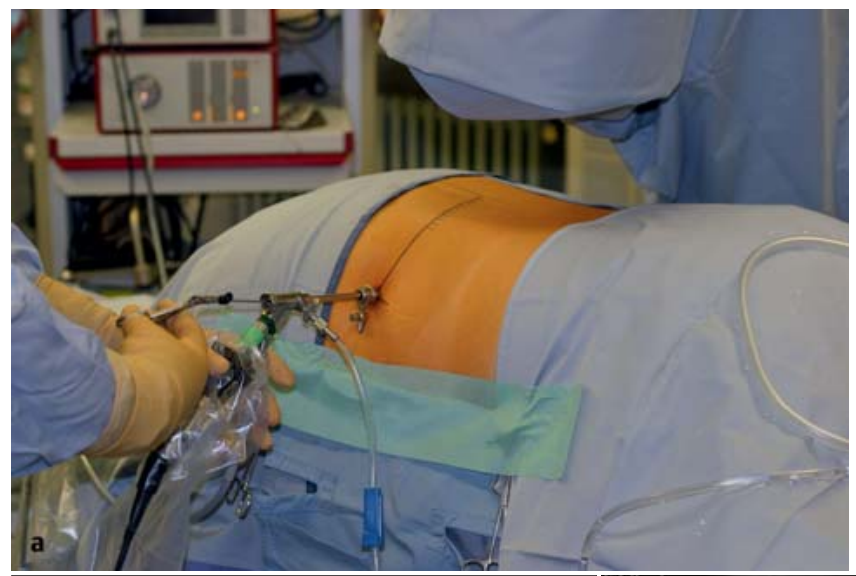

Abb. 3 a bis c Vollendoskopische transforaminale Technik mit lateralem Zugang an der Lendenwirbelsäule.

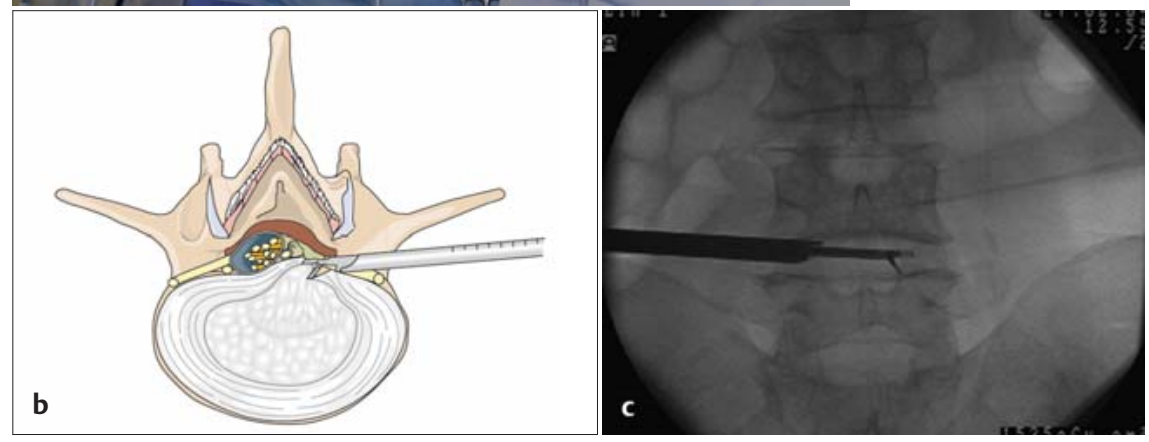

Aufgrund dieser Probleme wurde der vollendoskopische laterale transforaminale Zugang entwickelt, mit dem der Spinalkanal suffizient unter kontinuierlicher Visualisierung erreicht werden kann (Abb. 3). Das Arbeiten unter Spülflüssigkeit ergibt exzellente Sichtverhältnisse. Es erfolgt kein Abmessen in Zentimetern zur Festlegung eines Eintrittspunkts in die Haut, sondern eine individuelle anatomische Bestimmung unter radiografischer Kontrolle.

Bei der vollendoskopischen transforaminalen Technik muss ein möglichst lateraler Zugang gewählt werden, um ein suffizientes Erreichen des Spinalkanals unter Sicht zu gewährleisten.
Trotz des lateralen Zugangs und der Möglichkeit der Knochenresektion unter kontinuierlicher visueller Kontrolle haben sich während der Entwicklungsarbeit klar definierte Indikationen und damit auch Einschränkungen ergeben. Diese betreffen die Mobilität nach kranial und kaudal sowie die Verhinderung des Zugangs durch das Becken sowie Organe des Thorax und Abdomens [10,13, $14,16]$.

\section{Operative Technik} trans-/extraforaminaler Zugang

Die Operation wird in der Regel in Vollnarkose durchgeführt, was für den Patienten und Operateur komfortabler ist, 


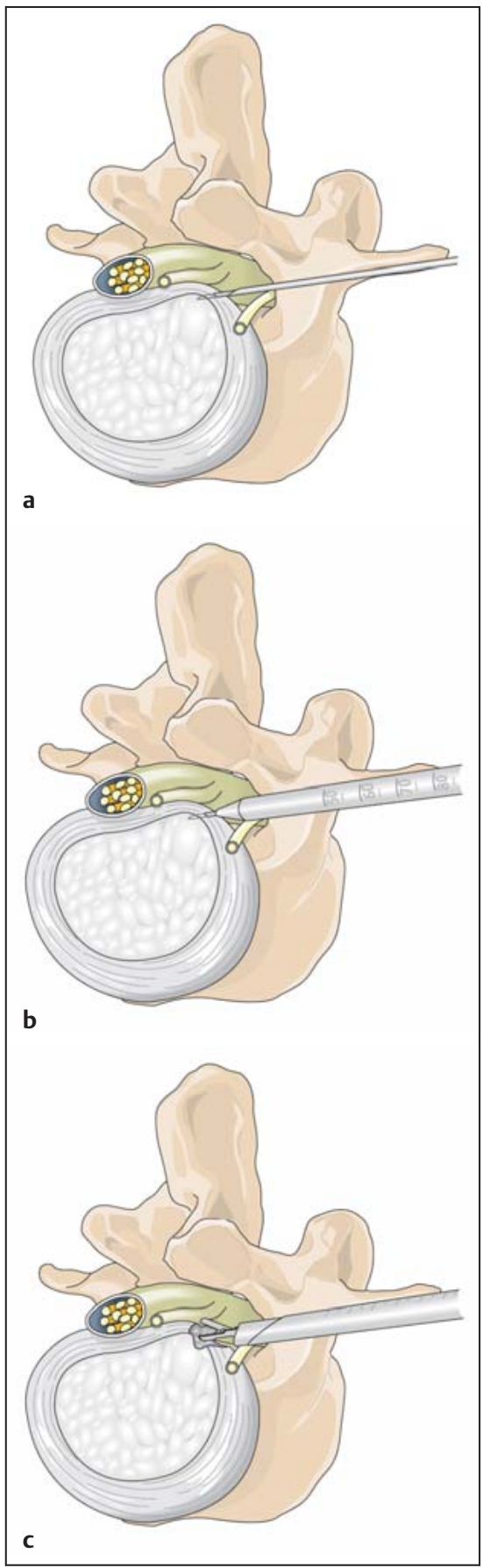

Abb.4a bis c Einbringen der Spinalkanüle (a), des Dilatators über den Zieldraht (b) und des Endoskops durch die Operationshülse (c) beim lateralen transforaminalen Zugang an der Lendenwirbelsäule.

bedarfsgerechte Lagerung ermöglicht und ausgedehntes Arbeiten innerhalb des Spinalkanals erlaubt. Sie erfolgt in Bauchlagerung auf einem röntgendurchlässigen Tisch unter orthograder radiologischer Kontrolle in 2 Ebenen. Zur Entlastung der Bauch- und Thoraxorgane wird der Patient im Hüft- und Thoraxbereich unterlagert. Der Operationstisch

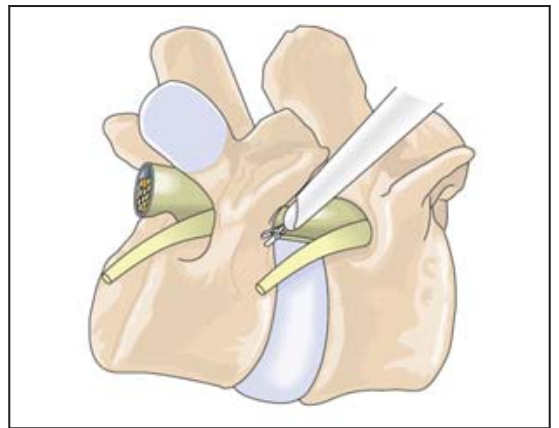

Abb.5 Der extraforaminale Zugang erfolgt direkt auf den kaudalen Pedikel.

ist in Abhängigkeit von Anatomie und Pathologie intraoperativ lordotisch oder kyphotisch einstellbar. Zur Infektionsprophylaxe wird eine Single-Shot-Antibiose appliziert.

Zu Beginn wird die Hautinzision lokalisiert. Operatives Ziel ist ein möglichst tangentiales Erreichen des Spinalkanals. In den Etagen L4/5 und L3/4 dient normalerweise im seitlichen Strahlengang die dorsale Linie des inferioren Gelenkfortsatzes als Grenze, die nicht nach ventral überschritten wird. Zur Vermeidung der Verletzung abdomineller Organe muss insbesondere in den kranialen Etagen oder bei nicht eindeutigem Befund zur Beurteilung und präoperativen Planung ein einzelner abdomineller CTScan durch die jeweilige Bandscheibe angefertigt werden. In Abhängigkeit hiervon ist ggf. ein individueller, weniger lateraler Zugang zu wählen.

Durch die Hautinzision wird eine atraumatische Spinalkanüle orthograd zum Bandscheibenraum in den Zielbereich eingebracht. Nach Einführen eines Zieldrahts und Entfernung der Kanüle erfolgt das Einbringen des kanülierten Dilatators. Der Zieldraht wird entfernt und über den Dilatator die Operationshülse vorgeschoben, in der Endposition mit der abgeschrägten Öffnung nach dorsal in Richtung neuraler Struktur. Ab hier wird die Dekompression unter Visualisierung und kontinuierlicher Spülung mit isotoner Kochsalzlösung durchgeführt. Weiteres erforderliches Eingehen in den Epiduralraum erfolgt unter Sicht (Abb. 4).

Erlaubt der knöcherne Durchmesser des Foramens keine Passage, wird das Foramen knöchern erweitert. Ist die Position des austretenden Nervs nicht eindeutig, wie z.B. bei intra- oder extraforaminalen Bandscheibenvorfällen oder bei Fora- menstenose, erfolgt ein extraforaminaler Zugang auf den kaudalen Pedikel als sichere Zone und die weitere Präparation Richtung Pathologie unter Sicht (Abb. 5). Auch bei Voroperation im Operationsbereich erfolgt der vollendoskopische Zugang in gleicher Weise. Die genaue Durchführung der Dekompression ist abhängig vom jeweiligen Befund. Nach Abschluss der Operation wird das Instrumentarium entfernt und die Stichinzision verschlossen, eine Drainage ist nicht erforderlich $[10,13,14,16]$.

\section{Interlaminärer Zugang}

Die vollendoskopische transforaminale Operation weist auch mit lateralem $\mathrm{Zu}$ gang Einschränkungen auf. Diese betreffen die Mobilität durch die knöchernen und neuralen Begrenzungen des Neuroforamens sowie die mögliche Verhinderung des Zugangs insbesondere in den unteren Etagen durch das Becken. Somit bleibt erfahrungsgemäß ein großes Spektrum an Pathologien, welches mittels des vollendoskopischen transforaminalen Vorgehens technisch nicht operiert werden kann.

Zur Reduktion operationsbedingter Traumatisierung der Strukturen des Spinalkanals ist die Ausnutzung anatomisch präformierter Zugangsbereiche sinnvoll. Neben dem Foramen intervertebrale existieren hier der Hiatus sacralis sowie das interlaminäre Fenster. Mittels der Epiduroskopie über den Hiatus sacralis ist die Resektion größerer Pathologien technisch nicht möglich. Somit bleibt der operative Zugang durch das interlaminäre Fenster, der in der lumbalen Wirbelsäulenchirurgie am längsten bekannt ist.

Aufgrund der Einschränkungen des transforaminalen Zugangs wurde der vollendoskopische interlaminäre Zugang entwickelt, der auch die Operation von Pathologien ermöglicht, die außerhalb des Indikationsspektrums des transforaminalen Vorgehens liegen (Abb. 6).

Die vollendoskopisch interlaminäre Technik ermöglicht auch die Operation von Pathologien, die transforaminal technisch inoperabel sind.

Das Erreichen des Spinalkanals über einen interlaminären Zugang mittels vollständiger oder partieller Laminektomie wurde seit Beginn des 20. Jahrhunderts beschrieben. Bereits 30 Jahre später wurden alternative Methoden zur 
Operation von Bandscheibenpathologien entwickelt. Optische Systeme werden seit den 80er-Jahren eingesetzt. In den 70er-Jahren wurde das mikrochirurgische Vorgehen mittels Einsatz des Mikroskops entwickelt, welches für interlaminäre Dekompressionen im Bereich des Spinalkanals den Status eines „Goldstandards“ erreichte. Über endoskopischassistierte Verfahren wird seit Ende der 90er-Jahre publiziert. Hierbei handelt es sich um die Visualisierung des eröffneten Operationssitus mittels eines Endoskops auf einen Monitor.

Zum operativen interlaminären Erreichen des Epiduralraums ist die Eröffnung des Spinalkanals erforderlich. Es muss ein ausreichender Zugang geschaffen werden, der Sicht in den Spinalkanal und das Arbeiten mit Instrumenten gewährleistet. Mögliche Probleme der Traumatisierung wurden bereits angesprochen. Das Mikroskop reduziert vornehmlich die Größe des Zugangswegs und schafft gute Sicht- und Lichtverhältnisse. Die Resektion der Strukturen des Spinalkanals kann meistens nicht vermieden werden. Der Zugang der endoskopisch-assistierten Technik kann schonender als das mikroskopische Vorgehen gestaltet werden. Der Vorteil liegt in der geringeren Distanz zwischen Arbeitsfeld und visualisierendem System. Sichtverhältnisse und Illumination sind meist schlechter. Heutzutage werden teilweise endoskopisch-assistierte Zugangstechnik und mikroskopisch-assistierte Operationstechnik kombiniert. Insgesamt muss bei allen Verfahren der Zugang meist größer gewählt werden, als es für das eigentliche Arbeiten im Spinalkanal notwendig wäre.

Beim vollendoskopischen interlaminären Zugang befindet sich das licht- und bildgebende System mit $25^{\circ}$-Blickwinkel direkt im jeweiligen Arbeitsbereich, sodass die Traumatisierung nicht nur im Zugangsweg, sondern auch an den Strukturen des Spinalkanals minimiert werden kann. Die $25^{\circ}$-Optiken bieten ein vergrößertes $360^{\circ}$-Sichtfeld. Durch Arbeiten unter kontinuierlichem Flüssigkeitsstrom bestehen exzellente Sichtverhältnisse, wie sie aus der arthroskopischen Chirurgie bekannt sind [9,10,13-15].

\section{Operative Technik}

interlaminärer Zugang

Auch hier wird die Operation in der Regel in Vollnarkose durchgeführt. Ebenfalls Bauchlagerung auf einem röntgen- durchlässigen Tisch unter orthograder radiologischer Kontrolle in 2 Ebenen. Zur Entlastung der Bauch- und Thoraxorgane wird der Patient im Hüft- und Thoraxbereich unterlagert. Der Operationstisch ist in Abhängigkeit von Anatomie und Pathologie intraoperativ lordotisch oder kyphotisch einstellbar. Zur Infektionsprophylaxe wird eine SingleShot-Antibiose appliziert.

Die Hautinzision erfolgt möglichst medial über dem interlaminären Fenster. Die kraniokaudale Lokalisation richtet sich nach dem Befund der jeweiligen Pathologie. Unter posterior-anteriorer Bildwandlerkontrolle wird der Dilatator stumpf auf den lateralen Rand des Lig. flavum oder auf die deszendierende Facette der Zygoapophysealgelenke eingebracht. Der weitere Ablauf erfolgt im seitlichen Strahlengang. Über den Dilatator wird die Operationshülse mit abgeschrägter Öffnung nach medial in Richtung Ligamentum flavum vorgeschoben. Ab hier erfolgt der weitere Eingriff unter Visualisierung und kontinuierlicher Spülung mit isotoner Kochsalzlösung.

Zum Erreichen des Spinalkanals wird das Lig. flavum lateral auf 3-5 mm inzidiert. Das weitere Eingehen wird durch die Elastizität des Ligaments ermöglicht. Die Operationshülse kann durch Rotation als 2. Instrument eingesetzt werden und dient z.B. durch Verlagerung der neuralen Strukturen nach medial als Nervenhaken (Abb. 7).

Erlaubt der knöcherne Durchmesser des interlaminären Fensters keine Passage, z.B. bei Operation einer Spinalkanalstenose oder bei deutlich dislozierten Sequestern, wird das Fenster knöchern erweitert. Bei Voroperation im Operationsbereich wird die mediale Kante der deszendierenden Facette aufgesucht und direkt entlang des Knochens nach ventral präpariert, bis die mediale Kante der aufsteigenden Facette lokalisiert werden kann. Hier erfolgt am Knochen das stumpfe Eingehen zum Grund des Spinalkanals. Zur Inspektion oder zu Arbeiten auf der kontralateralen Seite kann bei Bedarf über den gleichen Zugang „over the top“ die Gegenseite erreicht werden. Die genaue Durchführung der Dekompression ist abhängig vom jeweiligen Befund. Nach Abschluss der Operation wird das Instrumentarium entfernt und die Stichinzision verschlossen, eine Drainage ist nicht erforderlich $[9,10,13-$ 15].

\section{Optiken und Instrumentarien}

Die Stablinsenoptiken haben einen Durchmesser von $6,9 \times 5,6 \mathrm{~mm}$, eine Blickrichtung von $25^{\circ}$ und beinhalten einen exzentrisch gelegenen, intraendoskopischen Arbeitskanal von $4,1 \mathrm{~mm}$ Durchmesser. Die exzentrische Positionierung des Arbeitskanals ermöglicht zusätzliche Mobilität der Instrumente allein durch Drehen der Optik. Weiterhin sind in der Optik die Lichtleiter, das System des Flüssigkeitszustroms sowie das optische System selbst enthalten. Die Länge der Optik für den transforaminalen Zugang beträgt $207 \mathrm{~mm}$, für den interlaminären $165 \mathrm{~mm}$. Des Weiteren beinhalteten die Optiken die Anschlüsse für Lichtkabel und Kamera sowie einen $360^{\circ}$-drehbaren Anschluss für die Spülflüssigkeit. Der Ablauf der Spülflüssigkeit erfolgt über den Arbeitskanal sowie zwischen Optik und Operationshülse. Für eine temporäre Unterbrechung des Wasserabflusses durch den Arbeitskanal und damit für die Möglichkeit der Erhöhung des Spülwasserdrucks steht ein Membranaufsatz zur Verfügung.

Die Optiken werden frei durch Operationshülsen geführt, die einen Außendurchmesser von $7,9 \mathrm{~mm}$ haben und den jeweiligen Optiklängen angepasst sind. Die Operationshülsen weisen am Ende eine angeschrägte Öffnung auf, die ein Sicht- und Arbeitsfeld in einem Bereich ohne klar anatomisch-präformierte Höhlung gewährleisten. Eingebracht werden die Hülsen über einen Dilatator von 6,9 mm Außendurchmesser. Für den transforaminalen Zugang sind zusätzlich eine Spinalkanüle sowie ein Zieldraht erforderlich.

Es stehen verschiedene Instrumente mit Außendurchmessern von 2-4 mm zur Verfügung. Mit allen Instrumenten kann durch den intraendoskopischen Arbeitskanal, d.h. unter kontinuierlicher Sicht, gearbeitet werden. Die Art der Instrumente ist für beide Zugänge identisch, lediglich die jeweilige Länge ist denen der Optiken angepasst. Obwohl die Funktionen der Instrumente vergleichbar zu denen sind, die bei konventionellen Operationen eingesetzt werden, musste aufgrund der Miniaturisierung und den damit verbundenen mechanischen Besonderheiten eine komplette Neuentwicklung erfolgen. Zur Präparation, Koagulation und Sondierung kommt eine abwinkelbare, bipolare Kugelelektrode für radiofrequenten Strom zur Anwendung. Shaver- und Fräserköpfe haben 

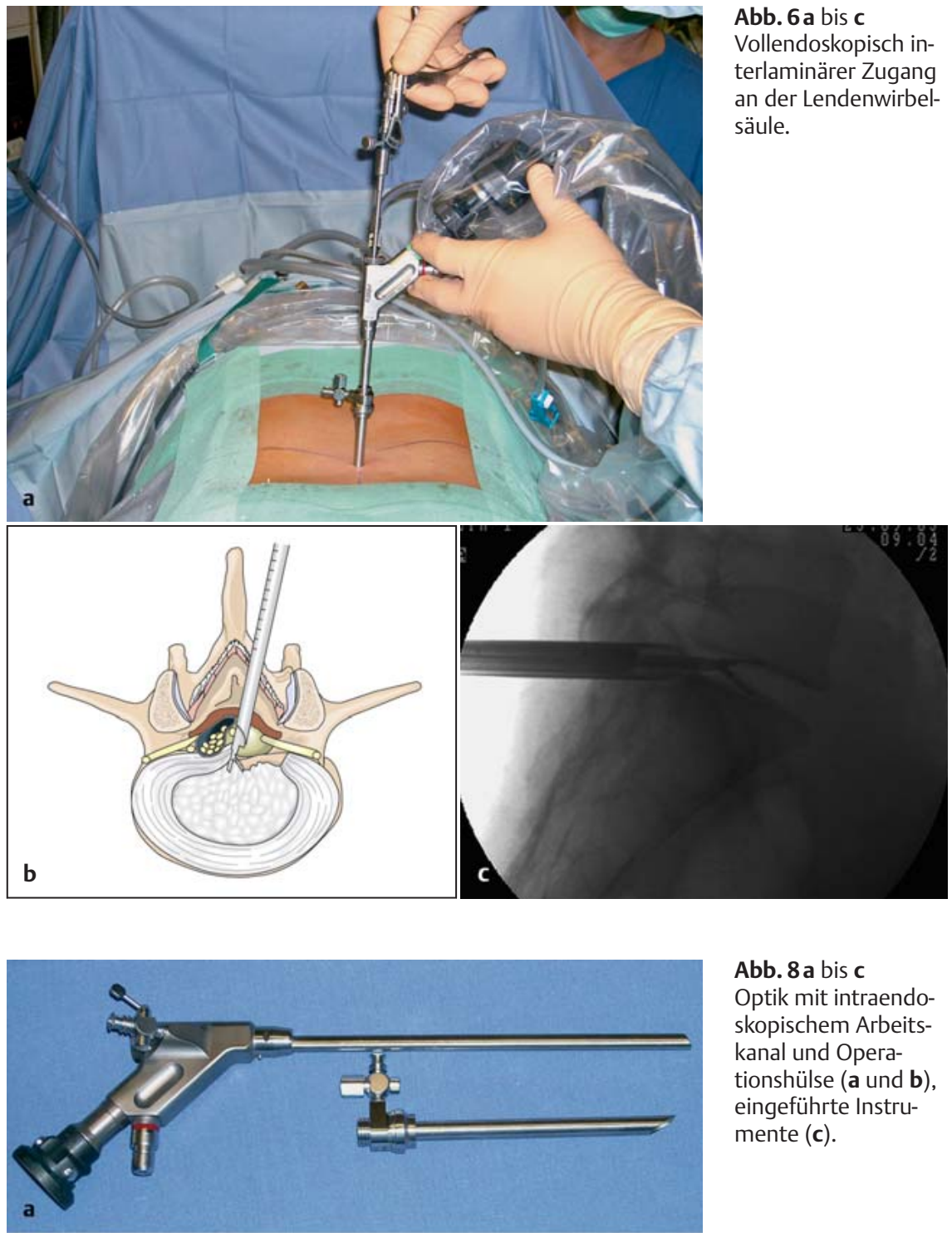

Abb. 8 a bis c Optik mit intraendoskopischem Arbeitskanal und Operationshülse (a und $\mathbf{b}$ ), eingeführte Instrumente (c).

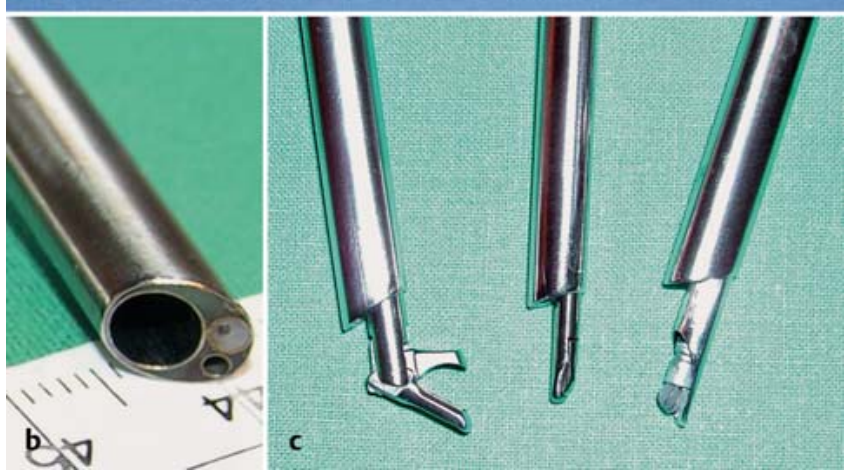

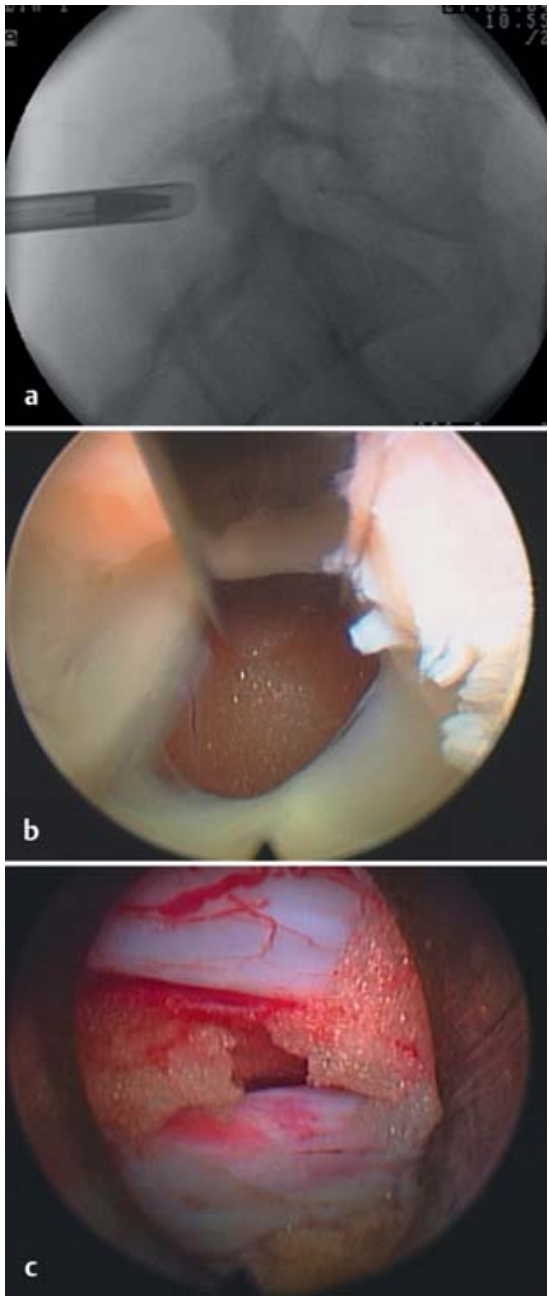

Abb. 7a bis c Einführen von Dilatator und Operationshülse (a), Eröffnung des Ligamentum flavum (b) und Präparation der neuralen Strukturen (c) beim interlaminären Zugang an der Lendenwirbelsäule.

schen Defiziten. Somit gelten als Haupteinschlusskriterien radikuläre Symptome im Bereich der unteren Extremitäten. Hauptausschlusskriterien sind reine Rückenschmerzen sowie Symptome aufgrund von Instabilität oder Deformität.

Hauptindikationen an der Lendenwirbelsäule sind radikuläre Symptome der unteren Extremitäten, d. h. eine Symptomatik in den Beinen und nicht im Rücken.

Eindeutige Indikationen für das vollendoskopische Vorgehen sind radikuläre Schmerzen bedingt durch folgende $\mathrm{Pa}$ thologien:

- sequestrierte oder nicht sequestrierte Bandscheibenvorfälle innerhalb des Spinalkanals

- sequestrierte oder nicht sequestrierte intra- und extraforaminale Bandscheibenvorfälle
Weiteren ist u.a. folgendes Zubehö 年, Operationstechniken benötigt wird oder eingesetzt werden kann: Monitor, Kameraeinheit, Lichtquelle mit Lichtkabel,
Shavermotor mit Steuerungseinheit, quenzgerät, Dokumentationssystem.

\section{Indikationen}

Die allgemeine Indikation zur Operation entspricht den heute gültigen Standards bei persistierenden oder intolerablen radikulären Schmerzen sowie neurologi- 


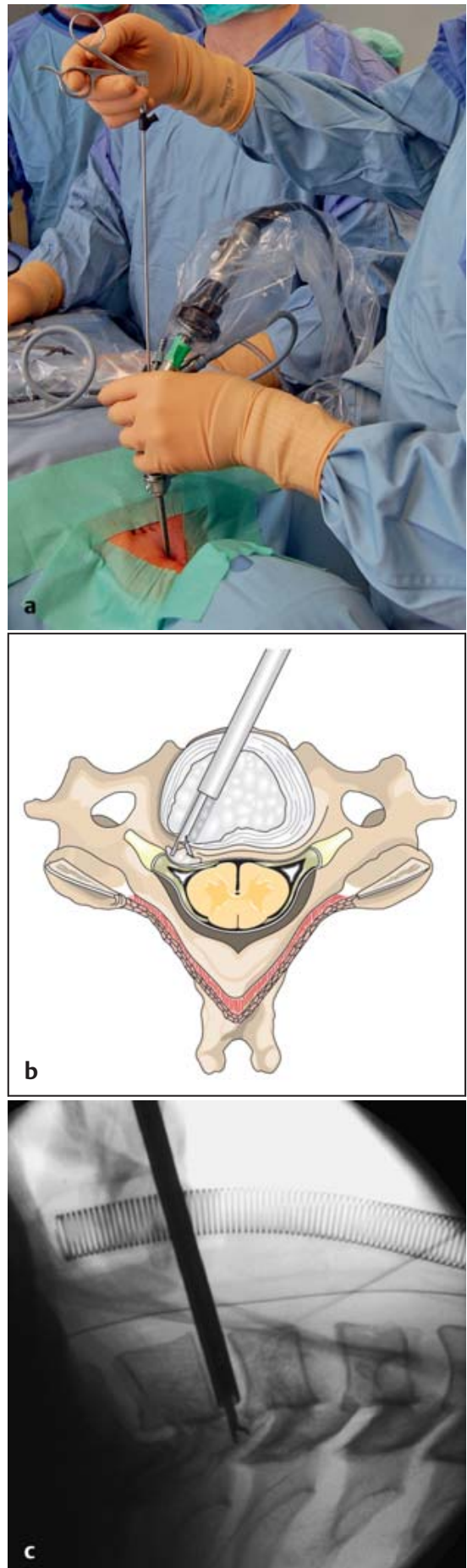

Abb.9a bis c Vollendoskopisch ventraler kontralateraler Zugang an der Halswirbelsäule.

- sequestrierte oder nicht sequestrierte Rezidivbandscheibenvorfälle jeglicher Lokalisation

- knöcherne und ligamentäre Spinalkanalstenosen

- Spinalkanalstenosen durch Zysten der Zygoapophysealgelenke

Generell kann der transforaminale $\mathrm{Zu}-$ gang als Verfahren der ersten Wahl gel- ten. Als Einschlusskriterien für den transforaminalen Zugang gelten alle intra- und extraforaminal gelegenen Pathologien. Bei Pathologien innerhalb des Spinalkanals sind folgende topografische Einschlusskriterien zu berücksichtigen: 1) Ausdehnung der Patholgie nach kranial bis maximal zum Beginn des darüberliegenden Pedikels, nach kaudal maximal bis zur Mitte des darunterliegenden Pedikels der jeweiligen Etage. 2) Im orthograden seitlichen Strahlengang Überdeckung der jeweiligen Etage durch das Becken bis maximal zur Mitte des darüberliegenden Pedikels. Zusätzlich darf der Zugangsweg nicht durch abdominelle Strukturen verlegt sein.

Als Einschlusskriterien für den interlaminären Zugang gelten alle innerhalb des Spinalkanals lokalisierten Indikationen, die aufgrund der genannten Kriterien transforaminal technisch inoperabel sind.

\section{Vollendoskopische Operationen der Halswirbelsäule}

\section{Ventraler kontralateraler Zugang}

In den 50er-Jahren wurden Operationsverfahren mit ventralem Zugang an der Halswirbelsäule beschrieben. Die sich hieraus entwickelnde ventrale Dekompression und Fusion ist heutzutage als „Goldstandard“ bei Dekompressionsoperationen anzusehen. Sie gilt als sicheres und suffizientes Verfahren mit guten Fusionsraten. Dennoch werden spezifische Probleme wie z.B. Sinterungen der Implantate, Pseudarthrosen oder Zugangskomplikationen beschrieben. Als besondere Nachteile der Fusion werden Anschlussdegenerationen diskutiert.

Um die Nachteile des Standardverfahrens zu reduzieren, wurden für das ventrale Vorgehen verschiedene Modifikationen beschrieben wie z. B. die ventrale Dekompression ohne Fusion, die ventrale Foraminotomie in verschiedenen Techniken oder die ventrale endoskopische transdiskale Dekompression. Die Bandscheibenprothese versucht die Rekonstruktion des Zwischenwirbelraums unter Erhalt der Segmentbeweglichkeit zu ermöglichen. Alle alternativen ventralen Techniken, mit Ausnahme der Bandscheibenprothese, beinhalten Dekompression ohne Fusion, d.h. ohne Rekonstruktion des Zwischenwirbelraums. Häufig wird versucht, die Bandscheibe so wenig wie möglich zu schädigen. Obwohl heutzutage die ventrale Dekom- pression und Fusion das Standardverfahren ist, werden die Fragen hinsichtlich progredienter segmentaler Sinterung oder Kyphosierung bei Operationstechniken ohne Fusion in der Literatur kontrovers diskutiert. Besonders unter Berücksichtigung von EBM-Kriterien gibt es keine einheitliche Aussage, die eindeutige Vorteile für eine zusätzliche Fusion erbringt.

Bei vollendoskopischen ventralen transdiskalen Operationen waren die beengten anatomischen Verhältnisse problematisch. Die geringe Höhe des Zwischenwirbelraums ließ nur kleine Optiken und Operationshülsen $\mathrm{zu}$, um eine zugangsbedingte Schädigung der Endplatten zu vermeiden. Hieraus resultierten technische Probleme wie z. B. schlechte Sichtverhältnisse, Arbeiten unter radiografischer Kontrolle ohne direkte Visualisierung oder eingeschränkte Knochenresektion.

Aufgrund dieser Probleme wurde ein neues Instrumentarium für den kontralateralen vollendoskopischen ventralen Zugang entwickelt, mit dem unter kontinuierlicher Spülung und Visualisierung mit $25^{\circ}$-Blickwinkel operiert werden kann (Abb.9). Dennoch bestehen aufgrund des transdiskalen Vorgehens eng definierte Indikationen [11].

\section{Operative Technik ventraler kontralateraler Zugang}

Die Operation wird in Vollnarkose in Rückenlage durchgeführt. Der Kopf wird fixiert, die Schulter ggf. nach unten gezogen, um auch in den unteren Etagen unter radiografischer Kontrolle arbeiten zu können. Die Halterung des Kopfes in der Mayfield-Klammer bietet gute Fixation und erlaubt auch bei unerwarteten Komplikationen den sofortigen Umstieg auf ein offenes Verfahren. Zur Infektionsprophylaxe wird eine Single-Shot-Antibiose appliziert.

Der Zugang erfolgt von kontralateral. Dies erfordert weniger Manipulation der Weichteile und erlaubt dem Operateur, mit den Instrumenten vorwärts und nicht rückwärts arbeiten zu können. Die ventrale Wirbelsäule wird palpiert, wobei Trachea und Ösophagus nach medial sowie der Gefäß-Nerven-Strang mit A. carotis und $V$. jugularis interna nach lateral manipuliert werden (Abb. 10).

Unter seitlicher Bildwandlerkontrolle wird der entsprechende Zwischenwir- 


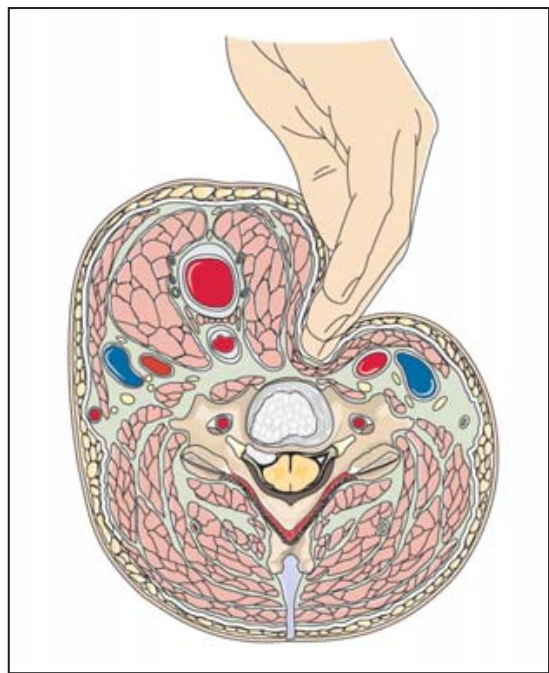

Abb. 10 Für den ventralen Zugang an der Halswirbelsäule muss zwischem dem GefäßNerven- und dem Eingeweidestrang die ventrale Wirbelsäule exakt palpiert werden.

belraum markiert und eine $5 \mathrm{~mm}$ Hautinzision am medialen Rand des M. sternocleidomastoideus durchgeführt. Unter kontinuierlicher Palpation der Wirbelsäule wird der Anfangsdilatator in den Zwischenwirbelraum eingebracht. Alternativ kann hier auch zunächst mit einer Spinalkanüle und nachfolgend mit dem Dilatator punktiert werden. Im posterior-anterioren Strahlengang wird die intradiskale Lage überprüft. Der weitere Ablauf erfolgt unter seitlicher Bildwandlerkontrolle. Über den Anfangsdilatator wird das kombinierte Operationshülsen-Dilatator-System in den Zwischenwirbelraum geschoben. Die Dilatatoren werden entfernt, die Operationshülse verbleibt mit der Öffnung dorsal im Zwischenwirbelraum. Ab hier erfolgt der weitere Eingriff unter Visualisierung und kontinuierlicher Spülung mit isotoner Kochsalzlösung (Abb. 11).

Auf der Seite der Pathologie werden dorsaler Anulus, dorsale Bereiche der Wirbelkörper oder Endplatten sowie der Processus uncinatus präpariert. Nachfolgend wird in Abhängigkeit von Pathologie und Anatomie das sequestrierte Bandscheibenmaterial dargestellt. Hierfür kann die Eröffnung des dorsalen Anulus und des hinteren Längsbands notwendig sein. Ebenso kann die Resektion von Knochen im dorsalen Bereich des kaudalen Wirbelkörpers oder des Processus uncinatus erforderlich werden.

In Abhängigkeit von der Präparation zeigt sich nach der Dekompression der ventrale Epiduralraum mit Dura des

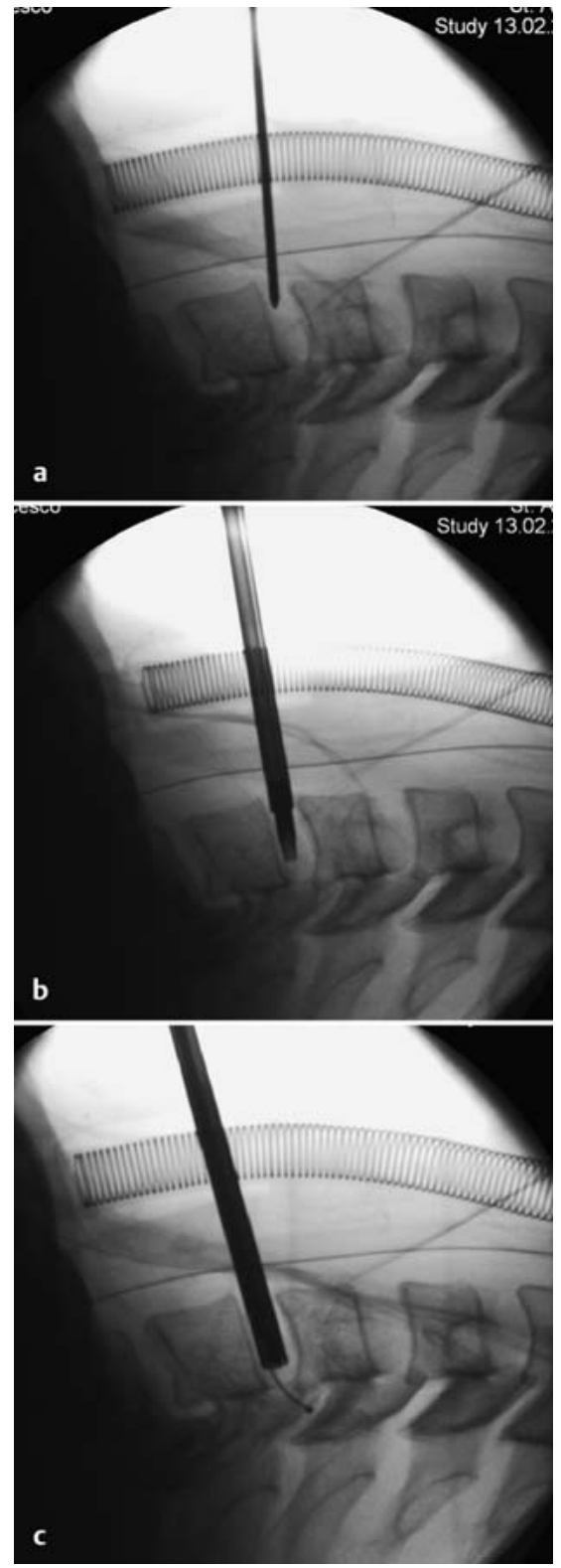

Abb. 11a bis c Einbringen des Anfangsdilatators (a), des Operationshülsen-Dilatator-Systems (b) und nachfolgend des Endoskops (c) beim ventralen Zugang an der Halswirbelsäule.

Myelons und dem Abgang des Spinalnervs. Unter Sicht werden die Operationshülse aus der Bandscheibe entfernt und freie Nukleusanteile reseziert. Weiteres Zurückziehen des Endoskops und Inspektion des Zugangswegs. Nach Entfernung des Instrumentariums Verschluss der Stichinzision, eine Drainage ist nicht erforderlich [11].

\section{Dorsaler Zugang}

In den 40er-Jahren wurde der dorsale Zugang zur Halswirbelsäule beschrieben. $\mathrm{Er}$ ist in veränderter Form bis heute neben der ventralen Dekompression und
Fusion das gebräuchlichste Alternativverfahren für Dekompressionsoperationen an der Halswirbelsäule. Zur Verminderung der Invasivität sind mikroskopisch-assistierte und endoskopisch-assistierte Techniken beschrieben, die heutzutage als sog. „Keyhole Foraminotomy" bezeichnet werden.

Unter Berücksichtigung entsprechender Kriterien kann die dorsale Operation bei Bandscheibenvorfall oder lateraler Stenose meist ohne zusätzliche Fusion durchgeführt werden. Als Nachteile werden zugangsbedingte Nackenschmerzen und intraoperative epidurale Blutungen beschrieben. Da das Myelon nicht manipuliert werden darf, ist die Foraminotomie nur bei lateralen Pathologien einsetzbar. Eine Rekonstruktion des Zwischenwirbelraums ist daher nicht möglich.

An der Halswirbelsäule findet sich im Gegensatz zur Lendenwirbelsäule im Spinalkanal das Myelon, d. h. zentrales Nervensystem, welches intraoperativ nicht manipuliert werden darf.

Aufgrund der Einschränkungen des ventralen Zugangs und der transdiskalen Technik wurde der vollendoskopische dorsale Zugang im Sinne einer vollendoskopischen Foraminotomie entwickelt (Abb. 12). Er ist dem interlaminären $\mathrm{Zu}$ gang der Lendenwirbelsäule ähnlich. Auch hier befindet sich das licht- und bildgebende System mit $25^{\circ}$-Blickwinkel direkt im jeweiligen Arbeitsbereich, sodass die Traumatisierung nicht nur im Zugangsweg, sondern auch an den Strukturen des Spinalkanals minimiert werden kann. Die $25^{\circ}$-Optiken bieten ein vergrößertes $360^{\circ}$-Sichtfeld. Durch Arbeiten unter kontinuierlichem Flüssigkeitsstrom bestehen exzellente Sichtverhältnisse. Auch der vollendoskopische dorsale Zugang ist nur bei lateralen Pathologien einsetzbar [12].

\section{Operative Technik \\ dorsaler Zugang}

Die Operation wird in Vollnarkose in Bauchlagerung durchgeführt. Der Kopf wird fixiert, die Schulter ggf. nach unten gezogen, um auch in den unteren Etagen unter radiografischer Kontrolle arbeiten zu können. Die Halterung des Kopfes in der Mayfield-Klammer bietet gute Fixation und erlaubt auch bei unerwarteten Komplikationen den sofortigen Umstieg auf ein offenes Verfahren. Die Halswirbelsäule wird entlordosiert. Zur Infek- 

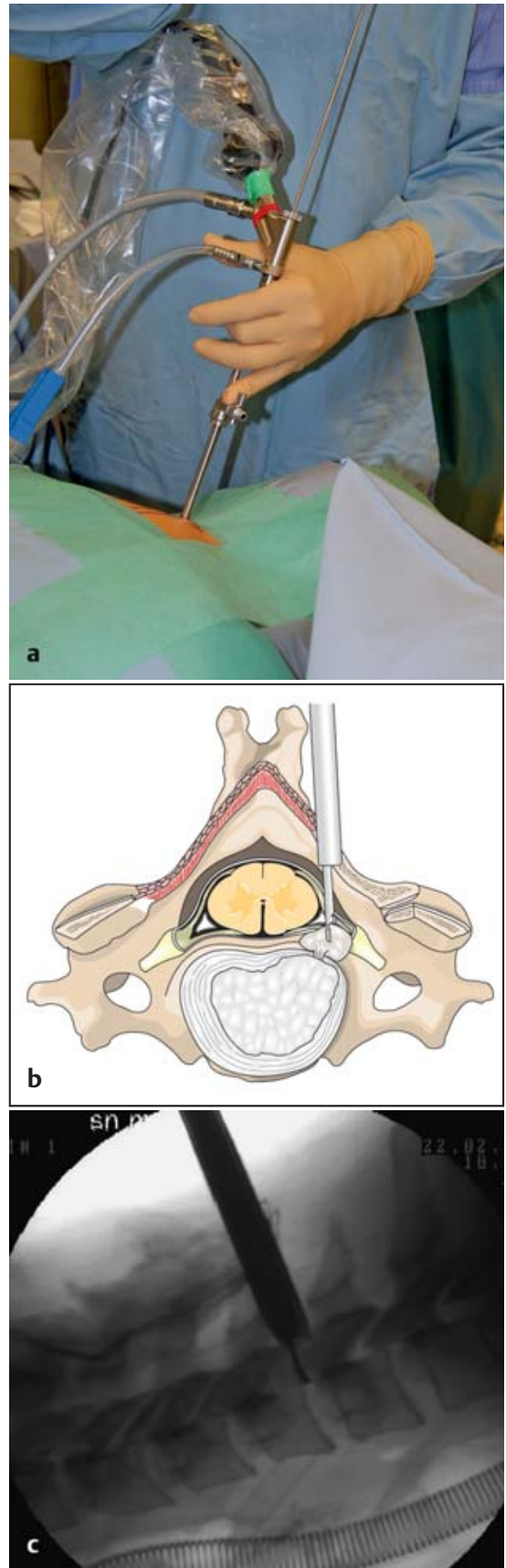

Abb.12a bis c Vollendoskopisch dorsaler Zugang an der Halswirbelsäule.

tionsprophylaxe wird eine Single-ShotAntibiose appliziert.

Zunächst wird unter posterior-anteriorer Bildwandlerkontrolle die Linie der Massa laterales auf der Haut markiert. Der weitere Ablauf erfolgt unter seitlichem Strahlengang. Bestimmung des Bandscheibensegments mit einer Kanüle auf der Linie der Massa laterales und Durchführung der Hautinzision. Der Dilatator wird stumpf auf die Massa lateralis bzw. das Zygoapophysealgelenk ein-
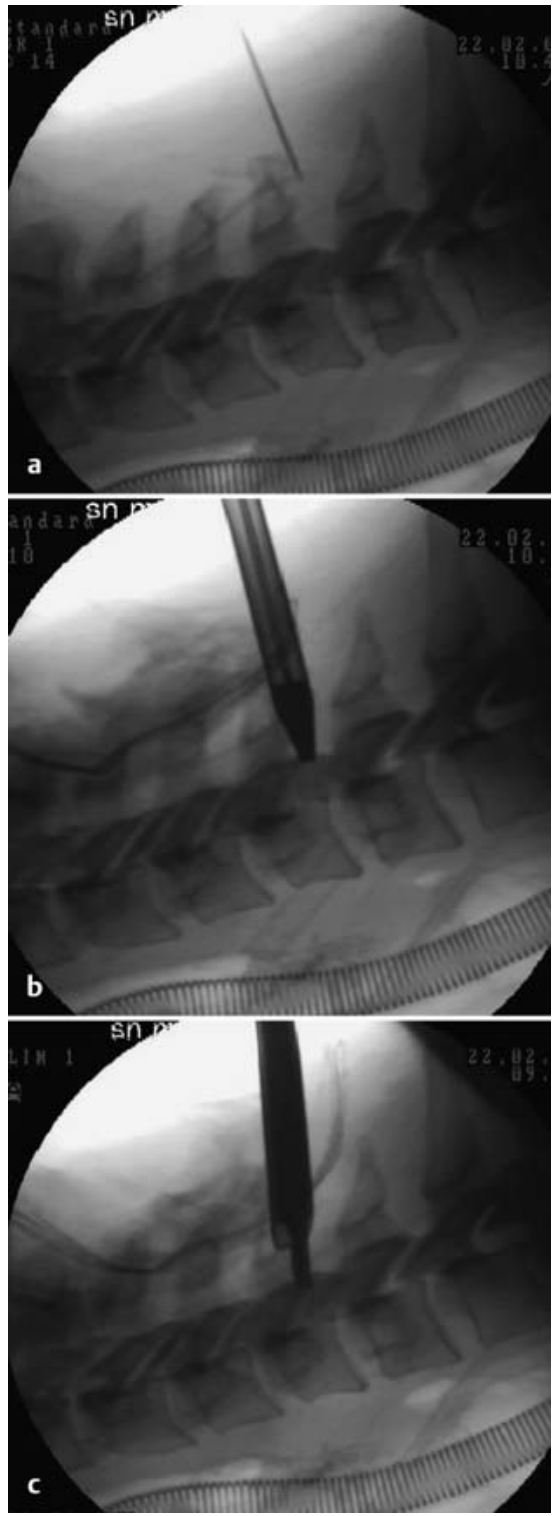

Abb. 13a bis c Markierung des Bandscheibenniveaus (a), Einbringen des Dilatators (b) und nachfolgend der Operationshülse (c) beim dorsalen Zugang an der Halswirbelsäule.

gebracht. Über den Dilatator wird die Operationshülse mit abgeschrägter Öffnung nach medial in Richtung Lig. flavum vorgeschoben und der Dilatator entfernt. Ab hier erfolgt der weitere Eingriff unter Visualisierung und kontinuierlicher Spülung mit isotoner Kochsalzlösung (Abb. 13).

Zunächst werden Gelenkanteile, kraniale und kaudale Laminakante sowie der interlaminäre Raum präpariert. Beginn der Foraminotomie mittels Knochenresektion an den medialen Gelenkanteilen sowie der kranialen und kaudalen Lamina. Nach Darstellung des Lig. flavum wird das Ligamentum lateral auf 3-5 mm
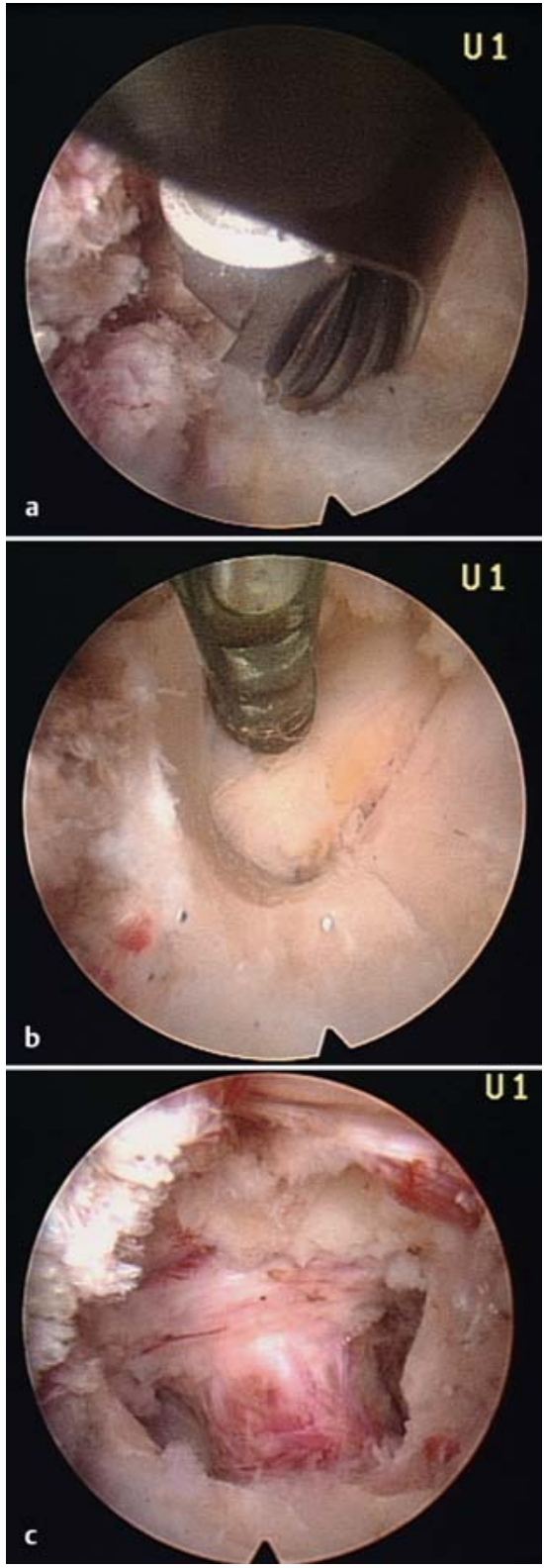

Abb. 14a bis c Foraminotomie mit Knochenresektion (a), Darstellung des Ligamentum flavum (b) und Präparation der neuralen Strukturen (c) beim dorsalen Zugang an der Halswirbelsäule.

inzidiert und der laterale Epiduralraum präpariert. Die laterale Begrenzung des zervikalen Myelons sowie der Abgang des Spinalnervs müssen eindeutig dargestellt werden (Abb. 14).

Die genaue Durchführung der Dekompression und Resektion sequestrierten Nukleusmaterials ist abhängig vom jeweiligen Befund. Nach Abschluss der Operation wird das Instrumentarium entfernt und die Stichinzision verschlossen, eine Drainage ist nicht erforderlich [12]. 


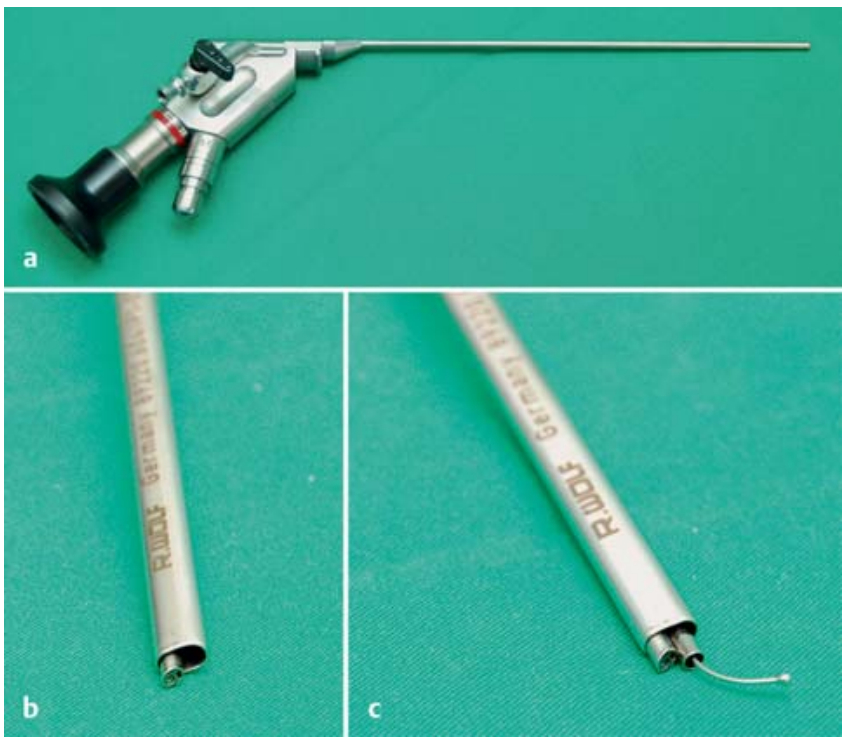

Abb. 15a bis c Optik (a) mit Operationshülse (b) und eingeführtem Tasthaken (c) für den ventralen Zugang an der Halswirbelsäule.

\section{Optiken und Instrumentarien}

Die Basis für den dorsalen Zugang bildete das System für den interlaminären Zugang an der Lendenwirbelsäule. Die kleineren Stablinsenoptiken mit einer Länge von $110 \mathrm{~mm}$ haben einen Durchmesser von $5,9 \times 5 \mathrm{~mm}$, ebenfalls eine Blickrichtung von $25^{\circ}$ sowie den exzentrisch gelegenen intraendoskopischen Arbeitskanal, der hier 3,1 mm Durchmesser aufweist. Auch hier sind in der Optik die Lichtleiter, das System des Flüssigkeitszustroms sowie das optische System selbst enthalten; des Weiteren die Anschlüsse für Lichtkabel und Kamera sowie ein $360^{\circ}$-drehbarer Anschluss für die Spülflüssigkeit. Der Ablauf der Spülflüssigkeit erfolgt ebenfalls über den Arbeitskanal sowie zwischen Optik und Operationshülse. Für eine temporäre Unterbrechung des Wasserabflusses durch den Arbeitskanal und damit für die Möglichkeit der Erhöhung des Spülwasserdrucks steht auch hier ein Membranaufsatz zur Verfügung.

Die Operationshülse ist der Optiklänge angepasst und hat einen Außendurchmesser von 6,9 mm. Auch hier weisen die Operationshülsen am Ende eine angeschrägte Öffnung auf, die ein Sichtund Arbeitsfeld in einem Bereich ohne klar anatomisch-präformierte Höhlung gewährleisten. Eingebracht werden die Hülsen über einen Dilatator von $5,9 \mathrm{~mm}$ Außendurchmesser.

Aufgrund der anatomisch räumlich eingeschränkten Verhältnisse im Zwischenwirbelraum, insbesondere in vertikaler Ausdehnung, musste für den ventralen transdiskalen Zugang an der Halswirbelsäule ein verändertes Konzept zur Anwendung kommen. Dies bedeutete die Entwicklung eines ovalen Systems, welches quer in den Zwischenwirbelraum eingebracht wird, somit eine geringere Höhe aufweist und die Endplatten der Bandscheibe schont. Die Stablinsenoptiken mit einer Länge von $150 \mathrm{~mm}$ haben eine Blickrichtung von $25^{\circ}$. Auch hier sind in der Optik die Lichtleiter, das System des Flüssigkeitszustroms sowie das optische System selbst enthalten; des Weiteren die Anschlüsse für Lichtkabel, Kamera und Spülflüssigkeit. Die Optik wird seitlich innerhalb der ovalen Operationshülse geführt. Der verbleibende Raum innerhalb der Operationshülse dient als Arbeitskanal. Somit konnte ein platzsparendes System entwickelt werden, was aber gleichzeitig den Nachteil geringerer Mobilität aufweist. Die Außendurchmesser der Operationshülsen betragen $3,8 \times 6,2 \mathrm{~mm}$ oder $4,1 \times 6,7 \mathrm{~mm}$, jeweils bei einer Länge von $102 \mathrm{~mm}$. Die Arbeitskanäle erlauben das Arbeiten mit Instrumenten bis $2,5 \mathrm{~mm}$ bzw. bis $3 \mathrm{~mm}$ Durchmesser. Der Ablauf der Spülflüssigkeit erfolgt zwischen Optik und Operationshülse. Die Operationshülse wird mit einem integrierten, herausnehmbaren Dilatator eingebracht, der wiederum über einen Anfangsdilatator von 1,8 mm Durchmesser geschoben wird (Abb. 15).

Vergleichbar zur Lendenwirbelsäule stehen für die zervikalen Zugänge verschiedene Instrumente mit Außendurchmessern von 2-3 mm zur Verfügung. Mit allen Instrumenten kann durch den intraendoskopischen Arbeitskanal, d.h. unter kontinuierlicher Sicht, gearbeitet werden. Die Art der Instrumente ist für beide Zugänge identisch, lediglich die jeweilige Länge ist denen der Optiken angepasst. Zur Präparation, Koagulation und Sondierung kommt eine abwinkelbare, bipolare Kugelelektrode für radiofrequenten Strom zur Anwendung. Shaver- und Fräserköpfe haben Außendurchmesser von 2,5-3 mm.

Wie an der Lendenwirbelsäule ist auch hier unter anderem folgendes Zubehör erforderlich, welches allgemein bei endoskopischen oder arthroskopischen Operationstechniken benötigt wird oder eingesetzt werden kann: Monitor, Kameraeinheit, Lichtquelle mit Lichtkabel, Shavermotor mit Steuerungseinheit, Pumpe für Spülflüssigkeit, Radiofrequenzgerät, Dokumentationssystem.

\section{Indikationen}

Die allgemeine Indikation zur Operation wird entsprechend den heute gültigen Standards bei persistierenden oder intolerablen radikulären Schmerzen sowie neurologischen Defiziten gestellt. Somit gelten als Haupteinschlusskriterien radikuläre Symptome im Bereich der oberen Extremitäten. Hauptausschlusskriterien sind reine Nackenschmerzen, Symptome aufgrund von Instabilität oder Deformität, isolierte Foramenstenosen sowie Symptome der Myelonkompression.

Eindeutige Indikationen für das vollendoskopische Vorgehen sind radikuläre Schmerzen bedingt durch weiche Bandscheibenvorfälle, die in kraniokaudaler Richtung maximal bis zur Hälfte des angrenzenden Wirbelkörpers sequestriert sind. Isolierte Foramenstenosen oder sog. „Bulging discs“ zeigen keine zufriedenstellende Ergebnisse und gelten nicht als Indikation.

Generell kann der dorsale Zugang als Verfahren der ersten Wahl gelten. Als Einschlusskriterien für den dorsalen $\mathrm{Zu}-$ gang gelten laterale Bandscheibenvorfälle, die mit ihrer Hauptmasse lateral der lateralen Myelonkante lokalisiert sind. Entscheidend ist die Vermeidung einer Manipulation des Myelons nach medial.

Für den ventralen Zugang gelten als Einschlusskriterien Bandscheibenvorfälle, die mit ihrer Hauptmasse medial der lateralen Myelonkante lokalisiert sind. Diese sind bei radikulären Symptomen eher selten. Die Operation lateraler Vorfälle von ventral kann durch den Processus uncinatus erschwert sein. Die ventrale 
Höhe des Zwischenwirbelraums muss mindestens $4 \mathrm{~mm}$ betragen. Ausschlusskriterium für den ventralen Zugang sind jegliche Voroperationen im Zugangsund Operationsgebiet. Durch Vernarbungen wird das Risiko der Verletzung von z.B. Ösophagus, Arterien oder Venen erhöht oder nicht mehr kontrollierbar.

Hauptindikation an der Halswirbelsäule sind radikuläre Symptome der oberen Extremitäten, d.h. eine Symptomatik in den Armen und nicht im Nacken, verursacht durch weiche Bandscheibenvorfälle. Der dorsale Zugang ist das Verfahren der 1 . Wahl.

\section{Vollendoskopische Operationen der Brustwirbelsäule}

An der Brustwirbelsäule sind Bandscheibenvorfälle oder degenerativ bedingte Stenosen, die zu konservativ nicht beherrschbaren radikulären Symptomen führen, sehr viel seltener als an den anderen Wirbelsäulenabschnitten. Die Notwendigkeit der Operation einer derart lateral gelegenen Pathologie gilt eher als Rarität. Somit erfolgte im Rahmen der Entwicklungsarbeit keine eigenständige Fokussierung auf die Brustwirbelsäule. Technisch können bei speziellen Voraussetzungen auch hier vollendoskopische Operationen durchgeführt werden, wobei der trans-/extraforaminale Zugang der Lendenwirbelsäule oder der dorsale Zugang der Halswirbelsäule jeweils mit modifiziertem Instrumentarium eingesetzt werden. In der Regel sind nur lateral gelegene Pathologien operabel, da aufgrund der Läsionsgefahr Manipulationen am Myelon vermieden werden müssen und ein lateraler transforaminaler Zugang durch die Thoraxorgane verhindert wird. Insgesamt besteht im Gegensatz zur Lendenwirbelsäule ein höheres Risiko der Verletzung neuraler und umgebender Strukturen sowie aufgrund dessen Einschränkungen in der Durchführung der Zugänge und des operativen Vorgehens. Unter Berücksichtigung der jeweiligen Anatomie, Pathologie und Symptomatik kann die Operation mittels konventionellem transthorakalen Vorgehen die einzige geeignete Option darstellen.

\section{Klinische Bewertung}

Das Ziel in der Entwicklung der operativen Therapie von radikulären Kompressionssyndromen durch Bandscheibenvorfälle oder Spinalkanalstenosen ist die suffiziente Dekompression unter opti- mierten Sichtbedingungen mit minimierter operationsinduzierter Traumatisierung und deren negativer konsekutiver Folgen. Bei der Einführung neuer Techniken müssen die klinischen Ergebnisse konventioneller Standardverfahren als Mindestkriterium erreicht werden [8]. Gleichzeitig sind Vorteile in Operationstechnik und/oder klinischen Variablen anzustreben.

Im Rahmen der klinischen Validierung der vollendoskopischen Techniken wurden immer die Möglichkeiten und Ergebnisse vergleichbarer etablierter Standardverfahren als Maßstab herangezogen. Hierfür wurden prospektive Nachuntersuchungsstudien und prospektiv randomisierte Vergleichstudien durchgeführt. Im Rahmen der Nachuntersuchungsstudien standen im Fokus die Fragestellungen hinsichtlich technischer Durchführung und deren Vor- und Nachteile, suffizienter Dekompression, möglicher Vorteile des reduzierten Operationstraumas und spezifischer Komplikationen. Für die Vergleichsstudien war das etablierte Kontrollverfahren im Bereich der Lendenwirbelsäule für die Indikationen primärer Bandscheibenvorfall, Rezidivbandscheibenvorfall und laterale Spinalkanalstenose jeweils die mikroskopisch-assistierte mikrochirurgische Dekompression; im Bereich der Halswirbelsäule für die Indikation Bandscheibenvorfall die mikroskopisch-assistierte ventrale Dekompression und Fusion [913].

\section{Lendenwirbelsäule}

Mögliche negative Folgen konventioneller Operationen an der Lendenwirbelsäule sind bekannt und zahlreich beschrieben $[1,4,5,7]$. Mittels des vollendoskopischen Vorgehens können im Literaturvergleich und in den zugrunde liegenden Studien Operationszeiten, Gewebetraumatisierung und Komplikationen reduziert werden. Dies entspricht publizierten Vorteilen eines minimalinvasiven intervertebralen und epiduralen Vorgehens. Die Möglichkeiten der Reduktion oder des Verzichts ossärer und ligamentärer Resektion sowie die atraumatischere Ausräumung des Zwischenwirbelraums können nach heutigem Wissensstand operationsinduzierte Instabilitäten vermeiden. Die Minimierung des Anulusdefekts, die mittels vollendoskopischer Technik möglich ist, scheint einen protektiven Einfluss $\mathrm{zu}$ haben. Operationsbedingte rehabilitative Maßnahmen sind nicht erforderlich. Es zeigt sich eine vergleichsweise hohe Rückkehr in das berufliche und sportliche Aktivitätsniveau. Bekanntermaßen erhöhte Morbidität bei Begleitfaktoren konnte nicht festgestellt werden. Die Rezidivrate bei Bandscheibenvorfällen zeigt im Vergleich zur Literatur und innerhalb der Studien keine signifikanten Unterschiede zum konventionellen Vorgehen. Revisionen können in gleicher Technik erfolgen. Die Art des Bandscheibenvorfalls und des Anulusdefekts scheinen größeren Einfluss auf die Rezidivrate zu haben als das Ausmaß der Ausräumung des Zwischenwirbelraums [3]. Insgesamt konnten keine relevanten Nachteile für die Anwendung der vollendoskopischen Technik zur Operation von Bandscheibenvorfällen, Rezidivbandscheibenvorfällen und Spinalkanalstenosen festgestellt werden. Gleichzeitig zeigten sich Vorteile in Operationstechnik und reduzierter Traumatisierung im Bereich des Zugangswegs und der Strukturen des Spinalkanals. Mikroskopisch-assistierte Dekompressionen erreichen gute klinische Ergebnisse zwischen 75 und 100\%. Mit den entwickelten vollendoskopischen Techniken können heutzutage klinisch übereinstimmende Ergebnisse wie mit dem konventionellen mikrochirurgischen Vorgehen erreicht werden. Die klinischen Verbesserungen sind für alle genannten Indikationen signifikant und konstant über die Nachuntersuchungszeiträume.

Die Entwicklung neuer Stablinsenoptiken mit großem intraendoskopischem Arbeitskanal und entsprechenden Instrumentarien hat die vollendoskopische Operation aller lumbalen Bandscheibenvorfälle inner- und außerhalb des Spinalkanals sowie von Spinalkanalstenosen technisch ermöglicht. In Kombination mit den neu entwickelten operativen Zugängen sind Parameter wie z.B. knöcherner Durchmesser des interlaminären Fensters und des Foramen intervertebrale oder Ausmaß der Sequestrierung von Bandscheibenmaterial nicht länger Kontraindikationen.

Um vollständige Dekompression sicher zu erreichen, müssen Bandscheibenvorfälle und Spinalkanalstenosen auch bei Verwendung einer vollendoskopischen Technik unter kontinuierlicher Visualisierung operiert werden. Mit Bezug auf den posterolateralen transforaminalen Zugang haben verschiedene Autoren die Entfernung von Sequestern aus dem Epiduralraum im Sinne einer retrograden Resektion von intradiskal durch den 
bestehenden Anulusdefekt beschrieben. Einige Publikationen stellen die Resektion aller Arten von Bandscheibenvorfällen dar. Dennoch ist die Operation besonders von innerhalb des Spinalkanals lokalisierten Pathologien limitiert. Die Entwicklung des lateralen transforaminalen Zugangs optimiert und ermöglicht den Zugang zum Spinalkanal und das Arbeiten unter kontinuierlicher Visualisierung. Probleme des posterolateralen Vorgehens werden hiermit beseitigt. Dennoch bestehen auch mit dem lateralen Zugang klare Ein- und Ausschlusskriterien und damit auch Einschränkungen. In Fällen, die technisch transforaminal nicht operabel sind, kann heutzutage der interlaminäre Zugang eingesetzt werden.

Das transforaminale Vorgehen ist aufgrund verminderter ossärer und ligamentärer Resektion als weniger traumatisierend und damit als Zugang der ersten Wahl zu bewerten. Aufgrund der anatomischen und pathologischen Voraussetzungen bestehen aber eindeutige Einschränkungen, sodass das interlaminäre Vorgehen das größere Spektrum aufweist $[9,13-16,19]$.

\section{Halswirbelsäule}

Mögliche Probleme des Standardverfahrens zur Operation zervikaler Bandscheibenvorfälle, der ventralen Dekompression und Fusion, sind bekannt und zahlreich beschrieben. Gleiches gilt für die gebräuchlichste Alternative, die dorsale „keyhole“ Foraminotomie.

Die guten klinischen Resultate der mikrochirurgischen ventralen Dekompression und Fusion, die als „Goldstandard“ gilt, können mit den vollendoskopischen Techniken heutzutage erreicht werden. Sie sind signifikant und konstant über die gesamten Nachuntersuchungszeiträume. Gleichzeitig sind Operationszeiten, Gewebetraumatisierung und Komplikationen reduziert. Dies entspricht den Erfahrungen mit minimalinvasivem intravertebralem und epiduralem Vorgehen im Bereich der Lendenwirbelsäule.

Operationsindizierte Nackenschmerzen oder Instabilitäten wurden nicht festgestellt. Beim dorsalen endoskopischen Vorgehen bleiben die Gelenkanteile nahezu vollständig erhalten. Auch beim ventralen endoskopischen Vorgehen trat keine Zunahme einer segmentalen Kyphose auf. Die mittlere Abnahme der Höhe des Zwischenwirbelraums war hier signifikant. Dies traf allerdings auch für die Kontrollgruppe mit Fusion zu. In der Literatur werden für diese Parameter höhere Werte beschrieben bei ventralen Dekompressionverfahren ohne Fusion [6]. Allerdings werden hier im Gegensatz zum endoskopischen Vorgehen Kürretierungen der Endplatten vorgenommen. Dies kann auch höhere spontane Fusionsraten erklären, die bei endoskopischer Technik nicht auftraten. Insgesamt konnte zwischen den genannten Parametern und dem klinischen Ergebnis kein Zusammenhang gefunden werden. Auch in der Literatur können die Fragen hinsichtlich progredienter Kyphosierung oder Instabilität nicht einheitlich nach EBM-Kriterien beantwortet werden [6]. Die Rezidivrate liegt innerhalb der Ergebnisse publizierter konventioneller Verfahren ohne Fusion. Revisionen können in gleicher Technik erfolgen. Im Gegensatz zu Verfahren mit Rekonstruktion des Zwischenwirbelraums sind Rezidivbandscheibenvorfälle technisch nicht auszuschließen.

Auch an der Halswirbelsäule hat die Entwicklung neuer und verschiedener Stablinsenoptiken und Instrumentarien die vollendoskopische Resektion von Bandscheibenvorfällen technisch ermöglicht. Insbesondere für den dorsalen Zugang ist suffiziente Knochenresektion Voraussetzung gewesen. Bei Anwendung des ventralen Zugangs schützt das modifizierte System mit ovaler Operationshülse Grund- und Deckplatten der Wirbelkörper. Wie im Bereich der Lendenwirbelsäule kann jeder Arbeitsschritt unter der erforderlichen, kontinuierlichen $\mathrm{Vi}$ sualisierung erfolgen.

Der dorsale endoskopische Zugang bietet keine speziellen Probleme. Er ist somit Zugang der ersten Wahl, aber nur für laterale Pathologien indiziert. Allerdings sind die meisten Bandscheibenvorfälle mit radikulärer Symptomatik in diesem Bereich lokalisiert. Der ventrale Zugang erfolgt dicht neben verletzungsanfälligen Strukturen. Während des Zugangs ist eine direkte Palpation der ventralen Wirbelsäule erforderlich. Diese kann bei muskulären Patienten in den kaudalen Etagen erschwert sein $[11,12]$.

Auch nach EBM-Kriterien erreichen vollendoskopische Dekompressionsoperationen mindestens die klinischen Resultate des konventionellen Standardverfahrens, gleichzeitig bestehen Vorteile in technischer, klnischer und ökonomischer Hinsicht.

\section{Vor- und Nachteile vollendoskopischer Operationen}

Basierend auf den operativen Erfahrungen und den klinischen Studien können folgende Vorteile der vollendoskopischen Techniken im Bereich der Lendenund Halswirbelsäule angeführt werden:

- Erleichterung für den Operateur durch gute Visualisierung, Ausleuchtung und erweitertes Sichtfeld durch $25^{\circ}$-Optiken

- kosteneffektives Verfahren durch kurze Operationszeiten, schnelle Rehabilitation und geringe postoperative Folgekosten

- reduzierte Traumatisierung

- erleichterte Revisionsoperationen

- Monitorbild als Ausbildungsgrundlage für Assistenten

- lumbal transforaminal Umgehung epiduraler Vernarbung beim Rezidivbandscheibenvorfall

- bei den zervikalen Zugängen Erhalt der Mobilität

Folgendes ist als nachteilig zu bewerten:

- Einschlusskriterien für die unterschiedlichen Zugänge müssen eingehalten werden

- eingeschränkte Möglichkeiten der Zugangserweiterung bei Problemen

- bis jetzt keine Möglichkeit der endoskopischen Duranaht

- schwierige Lernkurve

- lumbal transforaminal theoretisches Risiko der Verletzung des austretenden Nervs

- bei den zervikalen Zugängen nur direkte Dekompression und keine indirekte Dekompression durch Rekonstruktion des Zwischenwirbelraums

Gestützt auf die prospektiven, randomisierten, kontrollierten Studien ist auch unter Berücksichtigung von EBM-Kriterien zusammenfassend festzustellen, dass mittels der entwickelten vollendoskopischen Techniken bei den genannten Indikationen die zu den Standardverfahren gleichwertige suffiziente Dekompression erreicht wird mit reduzierter Traumatisierung, verbesserten Sichtbedingungen und positiver Kostenrelation. Besondere Aufmerksamkeit ist auf die jeweilige Indikation sowie die schwierige Lernkurve zu richten, die wie bei vielen neuen Verfahren zu Beginn ein erhöhtes Problem- und Risikopotenzial beinhalten kann.

Vollendoskopische Techniken sind operative Alternative und Erweiterung, dennoch werden konventionelle und maxi- 
malinvasive Verfahren im Gesamtkonzept der Wirbelsäulenchirurgie weiterhin benötigt.

\section{Fazit}

Vollendoskopische Operationen bieten Vorteile und sind als Erweiterung und Alternative innerhalb des Gesamtkonzepts der Wirbelsäulenchirurgie einzuordnen. Dennoch müssen Operateure weiterhin in der Durchführung konventioneller und offener Verfahren geübt sein, nicht nur um dem Patienten jeweilig ein geeignetes Therapiekonzept anbieten zu können, sondern auch, um mögliche Komplikationen vollendoskopischer Operationen zu beherrschen.

\section{Literatur}

${ }^{1}$ Abumi K, Panjabi MM, Kramer KM et al. Biomechanical evaluation of lumbar spinal stability after graded facetectomies. Spine 1990; 15: 1142-1147

2 Benini A. Lumbar spinal stenosis. An overview 50 years following initial description. Orthopade 1993; 6: 461-472

3 Carragee EJ, Han MY, Suen PW et al. Clinical outcomes after lumbar discectomy for sciata: the effects of fragment type and anular competence. J Bone Joint Surg $[\mathrm{Am}]$ 2003; 85 : 102-108

${ }^{4}$ Cooper R, Mitchell W, Illimgworth $K$ et al. The role of epidural fibrosis and defective fibrinolysis in the persistence of postlaminectomy back pain. Spine 1991; 16: 1044-1048
${ }^{5}$ Fritsch EW, Heisel J, Rupp S. The failed back surgery syndrome: reasons, intraoperative findings and long term results: a report of 182 operative treatments. Spine 1996; 21 : 626-633

${ }^{6}$ Hauerberg J, Kosteljanetz M, Boege-Rasmussen $T$ et al. Anterior cervical discectomy with or without fusion with Ray Titanium Cage. Spine 2008; 33: 458-464

${ }^{7}$ Kim SS, Michelsen CB. Revision surgery for failed back surgery syndrome. Spine 1992; 17: 957-960

${ }^{8}$ Maroon JC. Current concepts in minimally invasive discectomy. Neurosurgery 2002; 51 : 137-145

${ }^{9}$ Ruetten S, Komp M, Merk $H$ et al. Surgical treatment for lumbar lateral recess stenosis with the full-endoscopic interlaminar and transforaminal approach versus conventional microsurgical technique: a prospective, randomized, controlled study. J Neurosurg Spine 2009; 10: 476-485

${ }^{10}$ Ruetten S, Komp M, Merk H et al. Recurrent lumbar disc herniation following conventional discectomy: a prospective, randomized study comparing full-endoscopic interlaminar and transforaminal versus microsurgical revision. J Spinal Disord Tech 2009; 22: 122-129

11 Ruetten S, Komp M, Merk H et al. Full-endoscopic anterior decompression versus conventional anterior decompression and fusion in cervical disc herniations. Int Orthop 2008; DOI: 10.1007/s00264-008-0684-y

12 Ruetten S, Komp M, Merk $H$ et al. Full-endoscopic cervical posterior foraminotomy for the operation of lateral disc herniations using 5.9-mm endoscopes: a prospective, randomized, controlled study. Spine 2008; 33: 940948

13 Ruetten S, Komp M, Merk H et al. Full-endoscopic interlaminar and transforaminal lumbar discectomy versus conventional microsurgical technique: a prospective, randomized, controlled study. Spine 2008; 33: 931939
14 Ruetten S, Komp M, Merk H et al. Use of newly developed instruments and endoscopes: fullendoscopic resection of lumbar disc herniations via the interlaminar and lateral transforaminal approach. J Neurosurg Spine 2007; 6: $521-530$

15 Ruetten S, Komp M, Godolias G. A new full-endoscopic technique for the interlaminar operation of lumbar disc herniations using $6 \mathrm{~mm}$ endoscopes: prospective 2-year results of 331 patients. Minim Invasive Neurosurg 2006; 49: 80-87

16 Ruetten S, Komp M, Godolias G. An extreme lateral access for the surgery of lumbar disc herniations inside the spinal canal using the full-endoscopic uniportal transforaminal approach-technique and prospective results of 463 patients. Spine 2005; 30: 2570-2578

17 Schick U, Doehnert J, Richter A et al. Microendoscopic lumbar discectomy versus open surgery: an intraoperative EMG study. Eur Spine 2002; 11: 20-26

18 Torgerson WR, Dotter WE. Comparative roentgenographic study of the asymptomatic and symptomatic lumbar spine. J Bone Joint Surg 1976; 58: 850-853

19 Weber BR, Grob D, Dvorak J et al. Posterior surgical approach to the lumbar spine and its effect on the multifidus muscle. Spine 1997; 22: $1765-1772$

\section{Dr. med. Sebastian Ruetten} Leiter Ressort Wirbelsäulenchirurgie und Schmerztherapie

Zentrum für Orthopädie und Unfallchirurgie St. Anna-Hospital Herne Hospitalstraße 19 44649 Herne info@s-ruetten.com 